\title{
Model Selection Approaches for Predicting Future Order Statistics from Type II Censored Data
}

\author{
Jyun-You Chiang, ${ }^{1}$ Shuai Wang, ${ }^{1}$ Tzong-Ru Tsai $\left({ }^{2},{ }^{2}\right.$ and Ting $\mathrm{Li}^{3}$ \\ ${ }^{1}$ School of Statistics, Southwestern University of Finance and Economics, Chengdu, China \\ ${ }^{2}$ Department of Statistics, Tamkang University, New Taipei City, Taiwan \\ ${ }^{3}$ College of Management and Economics, Tianjin University, Tianjin, China
}

Correspondence should be addressed to Tzong-Ru Tsai; tzongru@gms.tku.edu.tw

Received 7 April 2018; Revised 4 July 2018; Accepted 14 August 2018; Published 8 October 2018

Academic Editor: Mohammed Nouari

Copyright (C) 2018 Jyun-You Chiang et al. This is an open access article distributed under the Creative Commons Attribution License, which permits unrestricted use, distribution, and reproduction in any medium, provided the original work is properly cited.

\begin{abstract}
This paper studies a discriminant problem of location-scale family in case of prediction from type II censored samples. Three model selection approaches and two types of predictors are, respectively, proposed to predict the future order statistics from censored data when the best underlying distribution is not clear with several candidates. Two members in the location-scale family, the normal distribution and smallest extreme value distribution, are used as candidates to illustrate the best model competition for the underlying distribution via using the proposed prediction methods. The performance of correct and incorrect selections under correct specification and misspecification is evaluated via using Monte Carlo simulations. Simulation results show that model misspecification has impact on the prediction precision and the proposed three model selection approaches perform well when more than one candidate distributions are competing for the best underlying distribution. Finally, the proposed approaches are applied to three data sets.
\end{abstract}

\section{Introduction}

For saving testing time and sample resource, censoring schemes often are considered to implement life tests. Type I censoring scheme and type II censoring scheme are two popular censoring schemes based on the criteria of test time censoring and failure number censoring. Plenty studies can be found for evaluating the reliability of lifetime components via using type I censoring test or type II censoring test. See examples like, [1-6] etc.

In this study, we mainly restrict our attention to using type II censoring scheme for predicting the censored sample for reliability evaluation when a discriminant problem is considered. In the type II censoring scheme, we consider an experiment where $n$ identical components are placed in the test simultaneously. Assuming that $r^{\text {th }}$ component fails, the experiment is terminated. Thus the last $(n-r)$ components are censored. In many engineering applications, censored data are not allowed for implementing statistical methods to obtain information. For example, if we like to conduct a factorial design or fractional factorial design based on the experimental design methods, most experimental design methods cannot be implemented with censored data. In such situation, a reliable procedure for predicting censored or unobserved observations is required. Moreover, if we can predict the unobserved observations and transform a censored data set into a complete data set, the parameter estimation problem becomes easy especially for dealing with the cases, which have no analytic solutions of the parameter estimators can be obtained. The purpose of predicting life length of the $s^{\text {th }}(r<s \leq n)$ item is equivalent to the life length of a $(n-s+1)$-out-of- $n$ system that was made up of $n$ identical components with independent life lengths. When $s$ $=n$, it is better known as the parallel system. For this issue, various methods have been developed to predict the censored data. Kaminsky and Nelson [7] provided interval and point prediction of order statistics. Fertig et al. [8] provided Monte Carlo estimates of the distribution percentiles to construct prediction intervals for samples from a Weibull or smallest extreme value distribution (SEV). Kaminsky and Rhodin 
[9] provided the maximum likelihood predictor (MLP) to predict the future order statistics and then estimate the unknown parameters. Wu et al. [10] proposed five new pivotal quantities to obtain prediction intervals of future order statistics from the Pareto distribution. Kundu and Raqab [11] describes the Bayesian inference and prediction of the two-parameter Weibull distribution. Panahi and Sayyareh [12] proposed parameter estimation and prediction of order statistics for the Burr type XII distribution. Some of these predictions are complex, or they need to construct complex statistical models. Therefore, these existing methods are not easy to apply.

In order to solve this problem, Raqab [13] modified the MLP method and proposed four modified MLPs (MMLPs) to predict the future order statistics for the normal distribution (ND). In order to simplify the estimation function, they considered four types of modification to approximate the terms of hazard rate and extended hazard rate functions form a ND, which has unknown mean and known standard deviation. Yang and Tong [14] used MMLP method to predict type II censored data from factorial experiments. They derived the simple explicit solutions for parameters for a ND, which has unknown mean and unknown standard deviation. Chiang [15] used another three MMLP procedures to predict type II censored data under the Weibull distribution. In his procedures, it is difficult to find the only root solution to the parameter estimation. However, the parameter estimation of MMLP method can be obtained via simple parameter explicit solution only in the ND. For other commonly used distributions, the likelihood equations of MMLP may be nonlinear and does not admit explicit solutions. Hence the parameter estimation of MMLP loses the advantage for other commonly used distributions.

Another important problem in life testing experiments is the model selection based on the existing sample. In practical applications, many statistical distributions are much alike, especially in censored data, and the underlying distribution of product quality characteristics is usually unknown. They may fit the data well in practical applications. However, their predictions may lead to a significant difference. Therefore, correctly identifying the underlying distribution is an important issue and it has long been studied. Dumonceaux and Antle [16] applied ratio of maximized likelihood (RML) to discriminating between the lognormal and Weibull distributions. Kundu and Manglick [17] proposed statistical methods to discriminate between the lognormal and gamma distributions. Kundu and Raqab [18] proposed a selection to discriminate between the generalized Rayleigh and lognormal distribution. Yu [19] provided a misspecification analysis method to discriminate between the ND and SEV for the design of experiment. Dey and Kundu [20] studied the discrimination problem between the lognormal and loglogistic distributions. Elsherpieny et al [21] considered the discrimination problem between the Weibull and log-logistic distributions. Ashour and Hashish [22] provided a numerical comparison study for using RML-procedure, S-procedure, and F-procedure in failure model discrimination. Pakyari [23] presented diagnostic tools based on the likelihood ratio test and the minimum Kolmogorov distance method to discriminate between the generalized exponential, geometric extreme exponential, and Weibull distributions. Elsherpieny et al. [24] provided a method to discriminate the gamma and log-logistic distributions based on progressive type II censored data. Although the inference methods in the aforementioned studies are valuable, the impacts of model misspecification on predicting the future order statistics have not been well studied.

Among the model discrimination problems, due to the well-developed theory and inferential procedures for the location-scale family of distributions, the model discrimination within the location-scale family of distributions is particularly important and it has received much attention. The main purpose of this paper is to address these issues and provide satisfactory estimators of parameters and predictors of future order statistics when the underlying distribution is unknown but it is a member in the location-scale family. Specifically, for lifetime analysis, the essence of this study is to predict the future order statistics for type II censored data when the underlying distribution is unknown but is a member of the location-scale family. The major contributions of this study for censored data prediction are presented in Figure 1.

The rest of this paper is organized as follows. Section 2 presents materials and methods. In this section, statistical methods to obtain approximate predictors for type II right censored variables are studied and two prediction methods are proposed to predict the type II right-censored variables based on the AMLEs. The ND and SEV are considered as the candidate distributions to compete the best distribution for obtaining the predictors of type II right-censored variables. In Section 3, we provide three algorithms to implement the three proposed model selection approaches to deal with the discrimination problem when obtaining the predictors of type II right-censored variables based on the proposed methods. An intensive simulation study is conducted in Section 4 to evaluate the performance of the proposed approaches. Then, three examples are used to demonstrate the applications of the proposed methodologies in Section 5. Some concluding remarks are provided in Section 6.

\section{Methods for Approximate Predictors}

2.1. Approximate Maximum Likelihood Estimation. Let $Y_{i}$ denote the failure time of $i^{\text {th }}$ item and $X_{i}=\log \left(Y_{i}\right)$, which follows a location-scale family, having the probability density function (PDF) and cumulative distribution function (CDF):

$$
f(x ; \mu, \sigma)=\frac{1}{\sigma} g\left(\frac{x-\mu}{\sigma}\right),
$$

and

$$
\begin{aligned}
F(x ; \mu, \sigma)=G( & \left.\frac{x-\mu}{\sigma}\right), \\
& -\infty<\mu<\infty, \sigma>0,-\infty<x<\infty,
\end{aligned}
$$

respectively, where $\mu$ is location parameter and $\sigma$ is scale parameter. $g(\cdot)$ and $G(\cdot)$ are the PDF and CDF of a member, 


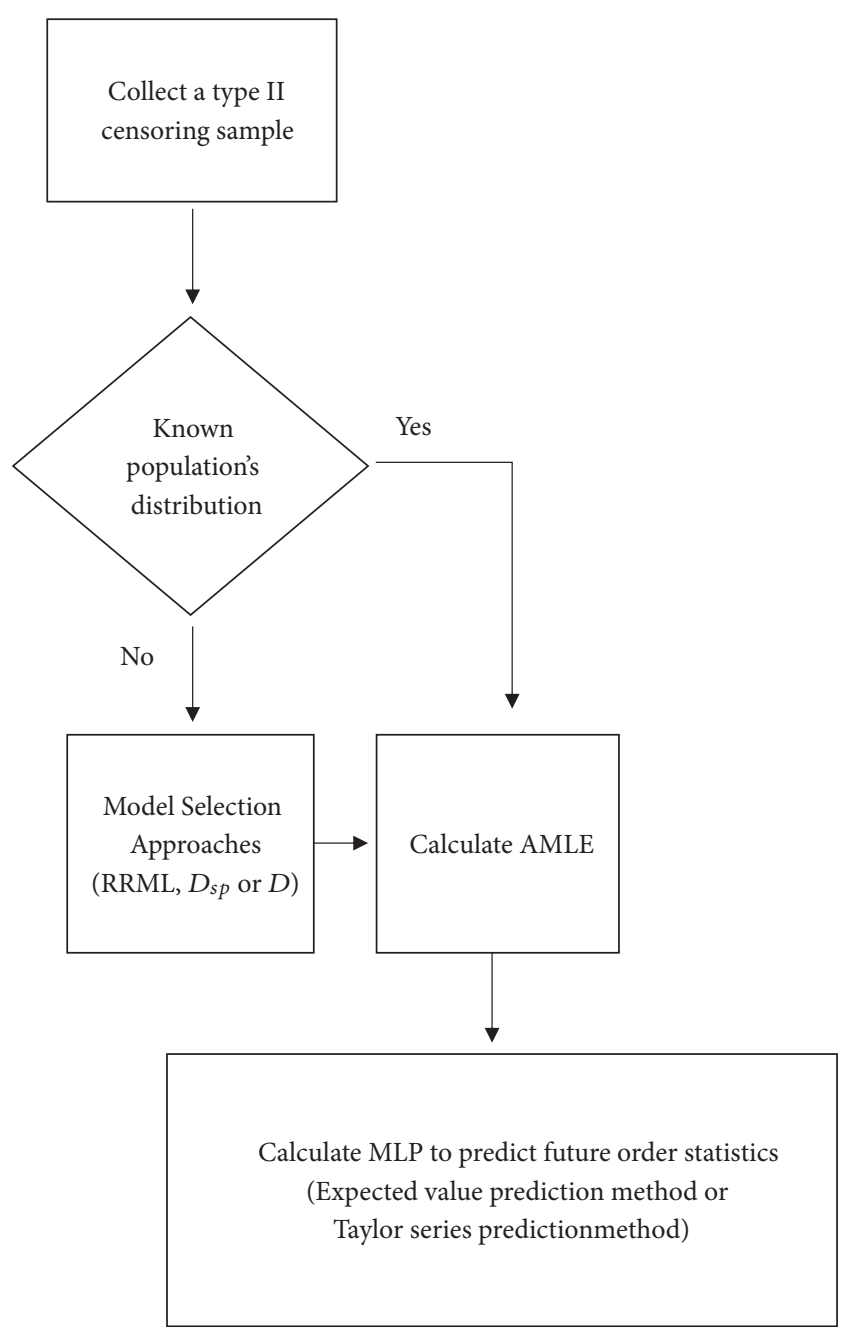

FIGURE 1: The flow chart of the major contribution of this study.

respectively, in the location-scale family. Denote the sample size by $n$, and denote type II censored sample with $r$ failures by $x_{1: n} \leq x_{2: n} \leq \cdots \leq x_{r: n}$, which are the realizations of $X_{1: n} \leq X_{2: n} \leq \cdots \leq X_{r: n}$, where $1 \leq r<s \leq n$. Our goal is to predict $x_{s: n}$ for $r<s \leq n$. Let $f(x) \equiv f(x ; \mu, \sigma)$ and $F(x) \equiv$ $F(x ; \mu, \sigma)$ here and after to simplify the notations. Kaminsky and Rhodin [9] considered prediction of $x_{s: n}$ having observed $\mathbf{x}=\left(x_{1: n}, x_{2: n}, \ldots, x_{r: n}\right)$, The predictive likelihood functions (PLF) of $X_{s: n}, \mu$ and $\sigma$ is

$$
\begin{aligned}
L & \left(X_{s: n}, \mu, \sigma ; \mathbf{x}\right) \equiv f\left(\mathbf{x}, X_{s: n} ; \mu, \sigma\right) \\
& =\frac{n !}{(s-r-1) !(n-s) !} \prod_{j=1}^{r} f\left(x_{j: n}\right) \\
& \cdot\left[F\left(X_{s: n}\right)-F\left(x_{r: n}\right)\right]^{s-r-1} f\left(X_{s: n}\right)\left[1-F\left(X_{s: n}\right)\right]^{n-s} .
\end{aligned}
$$

Please note that the capital notation $X_{s: n}$ in $F\left(X_{s: n}\right)$ is unknown and can be predicted based on the sample $\mathbf{x}$. Based on the proposed method by Raqab [13], the PLF of $X_{s: n}, \mu$ and $\sigma$ in (3) can be represented as a product of two likelihood functions, the PLF of $\mu$ and $\sigma$ (i.e., which is denoted as $L_{1}$ ) and the PLF of $X_{s: n}$ (i.e., which is denoted as $L_{2}$ ). Both likelihood functions are presented, respectively, by

$$
L_{1}(\mu, \sigma ; \mathbf{x})=\frac{n !}{(n-r) !} \prod_{j=1}^{r} f\left(x_{j: n}\right)\left[1-F\left(x_{r: n}\right)\right]^{n-r},
$$

and

$$
\begin{aligned}
L_{2}( & \left.X_{s: n} ; \mu, \sigma, \mathbf{x}\right) \\
= & \frac{(n-r) !}{(s-r-1) !(n-s) !} \frac{\left[F\left(X_{s: n}\right)-F\left(x_{r: n}\right)\right]^{s-r-1}}{\left[1-F\left(x_{r: n}\right)\right]^{n-r}} \\
& \times\left[1-F\left(X_{s: n}\right)\right]^{n-s} f\left(X_{s: n}\right) .
\end{aligned}
$$

In practice, we can obtain the MLEs of $\mu$ and $\sigma$, denoted by $\widehat{\mu}$ and $\widehat{\sigma}$, respectively, through maximizing $L_{1}(\mu, \sigma ; \mathbf{x})$ in (4). Then use $\widehat{\mu}$ and $\widehat{\sigma}$ to replace $\mu$ and $\sigma$ as the plug-in parameters in (5) to predict $X_{s: n}$. Let $z_{j: n}=\left(x_{j: n}-\mu\right) / \sigma$ for $j=1, \ldots, r, Z_{s: n}=\left(X_{s: n}-\mu\right) / \sigma$ for $s=r+1, \ldots, n$ and $\mathbf{z}=\left(z_{1: n}, z_{2: n}, \ldots, z_{r: n}\right)$, then we can rewrite (4) and (5) by

$$
L_{1} \equiv L_{1}(\mu, \sigma ; \mathbf{z})=C_{1} \prod_{j=1}^{r} \sigma^{-1} f\left(z_{j: n}\right)\left[1-F\left(z_{r: n}\right)\right]^{n-r}
$$

and

$$
\begin{aligned}
L_{2} & \equiv L_{2}\left(Z_{s: n} ; \widehat{\mu}, \widehat{\sigma}, \mathbf{z}\right)=C_{2} \sigma^{-1} \\
& \cdot \frac{\left[F\left(Z_{s: n}\right)-F\left(z_{r: n}\right)\right]^{s-r-1}}{\left[1-F\left(z_{r: n}\right)\right]^{n-r}}\left[1-F\left(Z_{s: n}\right)\right]^{n-s} \\
& \cdot f\left(Z_{s: n}\right),
\end{aligned}
$$

where $C_{1}=n ! /(n-r) !$ and $C_{2}=(n-r) ! /[(s-r-$ $1) !(n-s) !]$. After straightforward computations, the MLEs of $\mu, \sigma$ and $Z_{s: n}$ respectively can be obtained as the solutions of

$$
\begin{aligned}
& \frac{\partial \log \left(L_{1}\right)}{\partial \mu}=\frac{1}{\sigma}\left[\sum_{j=1}^{r} \Psi\left(z_{j: n}\right)+(n-r) h\left(z_{r: n}\right)\right]=0 \\
& \frac{\partial \log \left(L_{1}\right)}{\partial \sigma} \\
& =\frac{1}{\sigma}\left[-r+\sum_{j=1}^{r} \Psi\left(z_{j: n}\right) z_{j: n}+(n-r) h\left(z_{r: n}\right) z_{r: n}\right] \\
& =0
\end{aligned}
$$

and

$$
\begin{aligned}
\frac{\partial \log \left(L_{2}\right)}{\partial Z_{s: n}}= & (s-r-1) h_{1}\left(z_{r: n}, Z_{s: n}\right)-\Psi\left(Z_{s: n}\right) \\
& -(n-s) h\left(Z_{s: n}\right)=0,
\end{aligned}
$$


where

$$
\begin{aligned}
\Psi\left(Z_{j: n}\right)=- & \frac{f^{\prime}\left(Z_{j: n}\right)}{f\left(Z_{j: n}\right)}, \\
& j=1, \ldots, n, \text { where } Z_{j: n}=z_{j: n} \text { if } j \leq r, \\
h\left(Z_{j: n}\right)= & \frac{f\left(Z_{j: n}\right)}{1-F\left(Z_{j: n}\right)}, \\
& j=1, \ldots, n, \text { where } Z_{j: n}=z_{j: n} \text { if } j \leq r,
\end{aligned}
$$

and

$$
h_{1}\left(z_{r: n}, Z_{s: n}\right)=\frac{f\left(Z_{s: n}\right)}{F\left(Z_{s: n}\right)-F\left(z_{r: n}\right)} .
$$

Because of no analytic presentation for $\widehat{\mu}$ and $\widehat{\sigma}$, one needs to use numerical gradient computation methods, for example, the Newton-Raphson method, for obtaining $\widehat{\mu}$ and $\widehat{\sigma}$ via by equating (8) and (9). To obtain proper initial solutions for implementing gradient computation methods, we consider using the approximate MLEs (AMLE) of $\mu$ and $\sigma$ from Hossain and Willan [25] as their initial solutions in this study.

2.2. Approximate Maximum Likelihood Predictors. When we obtain the MLEs $\widehat{\mu}$ and $\widehat{\sigma}$, we can predict $X_{s: n}$ by using two approximation methods, the expected value prediction method and Taylor series prediction method. The resulting predictors of $X_{s: n}$ based on the expected prediction method is denoted by $\mathrm{MLP}_{\mathrm{E}}$, and the resulting predictors of $X_{s: n}$ based on the Taylor series prediction method is denoted by $\mathrm{MLP}_{\mathrm{T}}$. The two approximate methods mainly use two different methods to get the approximates of $h_{1}\left(z_{r: n}, Z_{s: n}\right)$ and $h\left(Z_{s: n}\right)$. Mehrotra and Nanda [26] proposed approximate maximum likelihood estimators for the ND and gamma distribution by replacing $h(x)$ and $x h(x)$ by their respective expected values and efficiencies compared to those for the best linear unbiased estimators for these distributions. Balakrishnan and Cohen [27] used the Taylor series expansion of $h(x)$ and $f(x) / F(x)$ at the points $F^{-1}\left(p_{s}\right)$ to obtain modified MLEs of the parameters of the ND and Rayleigh distribution, where $p_{i}=i /(n+1)$ for $i=1,2, \ldots, n$. The main point of their approach is that likelihood equations involve complicated terms and it is not possible to obtain an explicit form for MLE. So we follow their ideas and find an explicit form for the predictor of $X_{s: n}$.

Based on the expected value prediction method, replacing $(\mu, \sigma)$ with $(\widehat{\mu}, \widehat{\sigma})$, and replacing $h_{1}\left(z_{r: n}, Z_{s: n}\right)$ and $h\left(Z_{s: n}\right)$ by their respective expected values in (10). According to Raqab [13], the expected value of $f\left(Z_{j: n}\right), h_{1}\left(z_{r: n}, Z_{s: n}\right)$ and $h\left(Z_{s: n}\right)$ can be presented, respectively, by

$$
\begin{aligned}
E\left[f\left(Z_{j: n}\right)\right]=\frac{1}{n+1} \sum_{k=j+1}^{n+1} E\left[\Psi\left(Z_{k: n+1}\right)\right], & \\
& \quad \leq n \text { and } Z_{j: n}=z_{j: n} \text { if } j \leq r,
\end{aligned}
$$

$$
\begin{aligned}
E\left[h\left(Z_{j: n}\right)\right]=\frac{1}{n-j} \sum_{k=j+1}^{n} E\left[\Psi\left(Z_{k: n}\right)\right], & \\
& j \leq n-1 \text { and } Z_{j: n}=z_{j: n} \text { if } j \leq r,
\end{aligned}
$$

and

$$
\begin{aligned}
E\left[h_{1}\left(Z_{i: n}, Z_{j: n}\right)\right]= & \frac{1}{j-i-1} \sum_{k=j}^{n} E\left[\Psi\left(Z_{k: n}\right)\right], \\
& j-i \geq 2 \text {, and } Z_{j: n}=z_{j: n} \text { if } j \leq r .
\end{aligned}
$$

Based on the Taylor series prediction method, replacing $(\mu, \sigma)$ with $(\widehat{\mu}, \widehat{\sigma})$ and replacing $h\left(Z_{s: n}\right)$ and $h_{1}\left(Z_{r: n}, Z_{s: n}\right)$ with their Taylor series approximations at points $F^{-1}\left(p_{s}\right)$ and $\left(F^{-1}\left(p_{r}\right), F^{-1}\left(p_{s}\right)\right)$, respectively, in (10). In this study, we denote the $\mathrm{MLP}_{\mathrm{E}}$ and $\mathrm{MLP}_{\mathrm{T}}$ of $X_{s: n}$ under the candidate distribution $M$ by $\widehat{X}_{s: n}^{M, 1}$ and $\widehat{X}_{s: n}^{M, 2}$, respectively.

There are many common distributions in location-scale family of distributions. The widely used members including the ND, SEV, logistic distribution, etc. It is impossible to list all inference formulas for predicting $X_{s: n}$ under all widely used members in the location-scale family. In this study, we use ND and SEV as candidates to illustrating the applications of the proposed methods. But the suggested algorithms in this study can be applied for the cases with more than two candidate members. The reason to select the ND and SEV as candidates is due to the fact that the Weibull distribution and lognormal distribution are two widely used distributions for life testing applications. The Weibull and lognormal distributions can be respectively transformed into the SEV and ND by taking logtransformation.

If the underlying distribution is normal, the PDF of normal distribution is given by

$$
g(Z)=\phi(z)=\frac{1}{\sqrt{2 \pi}} e^{-z^{2} / 2} .
$$

Through using (17), we can obtain $\Psi(z)=-\phi^{\prime}(z) / \phi(z)=z$. The MLEs of normal distribution parameters are denoted by $\widehat{\mu}_{N}$ and $\widehat{\sigma}_{N}$. Replacing $\mu$ and $\sigma$ with $\widehat{\mu}_{N}$ and $\widehat{\sigma}_{N}$ in (6), we can represent (6) by

$$
\widehat{L}_{N}\left(\widehat{\mu}_{N}, \widehat{\sigma}_{N}\right)=C_{1} \prod_{j=1}^{r} \widehat{\sigma}_{N}^{-1} \phi\left(z_{j: n}\right)\left[1-\Phi\left(z_{r: n}\right)\right]^{n-r},
$$

where $\Phi(\cdot)$ is the CDF of the standard ND. According to (15) and (16), $h_{1}\left(z_{r: n}, Z_{s: n}\right)$ and $h\left(Z_{s: n}\right)$ can be replaced with their respective expected values in (10). Equation (10) can be rewritten as

$$
E\left(Z_{s: n}\right)-\widehat{Z}_{s: n}=0 .
$$

The values of $E\left(Z_{j: n}\right)$ are available and have been tabulated by Teichroew [28]. Hence, $M P_{\mathrm{E}}$ of $X_{s: n}$ for ND can be derived as

$$
\widehat{X}_{s: n}^{N, 1}=\widehat{\mu}_{N}+\widehat{\sigma}_{N} E\left(Z_{s: n}\right) .
$$


Because $E\left(Z_{s: n}\right) \geq z_{r: n}$ is a necessary condition, we modify (20) by

$$
\widehat{X}_{s: n}^{N, 1}=\max \left\{\widehat{\mu}_{N}+\widehat{\sigma}_{N} E\left(Z_{s: n}\right), x_{r: n}\right\}
$$

and use $\widehat{X}_{s: n}^{N, 1}$ in (21) to protect $X_{s: n}$ for $r+1 \leq s \leq n$.

Based on the Taylor series prediction method, the functions $h\left(Z_{s: n}\right)$ and $h_{1}\left(z_{r: n}, Z_{s: n}\right)$ are expanded by using the Taylor series around points $F^{-1}\left(p_{s}\right)$ and $\left(F^{-1}\left(p_{r}\right), F^{-1}\left(p_{s}\right)\right)$, respectively. According to Raqab [13], we can approximate $h\left(Z_{s: n}\right)$ and $h_{1}\left(z_{r: n}, Z_{s: n}\right)$ by

$$
h\left(Z_{s: n}\right)=\frac{f\left(Z_{s: n}\right)}{1-F\left(Z_{s: n}\right)} \approx \alpha+\beta Z_{s: n},
$$

and

$$
\begin{aligned}
h_{1}\left(z_{r: n}, Z_{s: n}\right) & =\frac{f\left(Z_{s: n}\right)}{F\left(Z_{s: n}\right)-F\left(z_{r: n}\right)} \\
& \approx \gamma+\rho z_{r: n}-v_{s} Z_{s: n} .
\end{aligned}
$$

The values of $\alpha, \beta, \gamma, \rho$ and $v_{s}$ are given in Appendix A. Equation (10) can be rewritten by

$$
\begin{gathered}
(s-r-1)\left(\gamma+\rho z_{r: n}-v_{s} Z_{s: n}\right)-z_{s: n} \\
-(n-s)\left(\alpha+\beta Z_{s: n}\right)=0 .
\end{gathered}
$$

The $\mathrm{MLP}_{\mathrm{T}}$ of $X_{s: n}$ can be obtained by

$$
\begin{gathered}
\widehat{X}_{s: n}^{N, 2}=\max \left\{\frac{(s-r-1) \rho x_{r: n}}{(s-r-1) v_{s}+1+(n-s) \beta}\right. \\
+\left[1-\frac{(s-r-1) \rho}{(s-r-1) v_{s}+1+(n-s) \beta}\right] \widehat{\mu}_{N} \\
\left.+\frac{(s-r-1) \gamma-(n-s) \alpha}{(s-r-1) v_{s}+1+(n-s) \beta} \widehat{\sigma}_{N}, x_{r: n}\right\},
\end{gathered}
$$

where $r+1 \leq s \leq n$.

If the underlying distribution is SEV, the PDF of the SEV is given by

$$
g(z)=\phi_{\text {sev }}(z)=e^{z-e^{z}}
$$

Based on the expected value prediction method, $\Psi(z)=$ $-\phi_{\text {sev }}^{\prime}(z) / \phi_{\text {sev }}(z)=e^{z}-1$. Using (8) and (9), the MLEs of $\mu$ and $\sigma$ are denoted by $\widehat{\mu}_{S}$ and $\widehat{\sigma}_{S}$, respectively. Replacing $\mu$ and $\sigma$ with $\widehat{\mu}_{S}$ and $\widehat{\sigma}_{S}$ in (6), (6) can be represented by

$$
\widehat{L}_{S}\left(\widehat{\mu}_{S}, \widehat{\sigma}_{S}\right)=C_{1} \prod_{j=1}^{r} \widehat{\sigma}_{S}^{-1} \phi_{s e v}\left(z_{j: n}\right)\left[1-\Phi_{s e v}\left(z_{r: n}\right)\right]^{n-r},
$$

where $\Phi_{\text {sev }}(z)=1-\exp [-\exp (z)]$ is the CDF of the standard SEV. Then $h_{1}\left(z_{r: n}, Z_{s: n}\right)$ and $h\left(Z_{s: n}\right)$ are replaced with their respective expected values in Eq. (10). Equation (10) can be rewritten as

$$
\begin{gathered}
(s-r-1) E\left[h_{1}\left(z_{r: n}, Z_{s: n}\right)\right]-\left(e^{\widehat{Z}_{s: n}}-1\right) \\
-(n-s) E\left[h\left(Z_{s: n}\right)\right]=0 .
\end{gathered}
$$

The $\mathrm{MLP}_{\mathrm{E}}$ of $X_{s: n}$ can be obtained as

$$
\widehat{X}_{s: n}^{S E V, 1}=\max \left\{\widehat{\mu}_{S}+\widehat{\sigma}_{S} \ln \left(E\left[\Psi\left(Z_{s: n}\right)\right]+1\right), x_{r: n}\right\}
$$

for $r+1 \leq s \leq n$ and $E \Psi\left(Z_{s: n}\right)=E\left(e^{Z_{s: n}}-1\right)$.

Based on the Taylor series prediction method, expanding $h\left(Z_{s: n}\right)$ and $h_{1}\left(z_{r: n}, Z_{s: n}\right)$ by using the Taylor series at the points $F^{-1}\left(p_{s}\right)$ and $\left(F^{-1}\left(p_{r}\right), F^{-1}\left(p_{s}\right)\right)$, respectively. We obtain

$$
h\left(Z_{s: n}\right)=\frac{f\left(Z_{s: n}\right)}{1-F\left(Z_{s: n}\right)} \approx 1-\alpha_{s}-\beta_{s} Z_{s: n}
$$

and

$$
\begin{aligned}
h_{1}\left(z_{r: n}, Z_{s: n}\right) & =\frac{f\left(Z_{s: n}\right)}{F\left(Z_{s: n}\right)-F\left(z_{r: n}\right)} \\
& \approx \gamma_{E}+\rho_{E} z_{r: n}+v_{E} Z_{s: n} .
\end{aligned}
$$

The values of $\alpha_{s}, \beta_{s}, \gamma_{E}, \rho_{E}$ and $v_{E}$ are given in Appendix B. Equation (10) can be rewritten as

$$
\begin{gathered}
(s-r-1)\left(\gamma_{E}+\rho_{E} z_{r: n}+v_{E} Z_{s: n}\right)-e^{Z_{s: n}}-1 \\
-(n-s)\left(1-\alpha_{s}-\beta_{s} Z_{s: n}\right)=0
\end{gathered}
$$

The $\mathrm{MLP}_{\mathrm{T}}$ of $X_{s: n}$ can be derived as

$$
\begin{aligned}
& \widehat{X}_{s: n}^{S E V, 2}=\max \left\{\frac{-(s-r-1) v_{E} x_{r: n}}{(s-r-1) \rho_{E}+\beta_{s}+(n-s) \beta_{s}}\right. \\
& +\left[1+\frac{(s-r-1) v_{E}}{(s-r-1) \rho_{E}+\beta_{s}+(n-s) \beta_{s}}\right] \widehat{\mu}_{S} \\
& \left.-\frac{(s-r-1) \gamma_{E}+\alpha_{s}-(n-s)+(n-s) \alpha_{s}}{(s-r-1) \rho_{E}+\beta_{s}+(n-s) \beta_{s}} \widehat{\sigma}_{S}, x_{r: n}\right\},
\end{aligned}
$$

for $r+1 \leq s \leq n$.

\section{Three Model Selection Approaches}

When several candidate distributions are competing for the best underlying distribution and the users cannot identify which one distribution is the best, we suggest three approaches to discriminate the candidate distributions, the ratio of the maximized likelihood (RRML) approach, modification $D_{S P}$ approach (shorted as $D_{S P}$ approach), and modification $D$ approach (shorted as the $D$ approach), to obtain the predictor of $\widehat{X}_{s: n}$. It is noticed that the idea of the $D_{S P}$ approach and $D$ approach is based on goodness-of-fit test methods. All these three approaches can be implemented to obtain the predictor of $X_{s: n}$ via using Algorithms 1-3.

Algorithm 1 (the RRML approach).

Step 1. Collect a type II censored sample, which has size $n$ and $r$ observed failure times; we consider $k$ candidate distributions.

Step 2. Obtain $\left(\widehat{\mu}_{M_{i}}, \widehat{\sigma}_{M_{i}}\right)$ and $\widehat{L}_{M_{i}}\left(\widehat{\mu}_{M_{i}}, \widehat{\sigma}_{M_{i}}\right)$ for the candidate distribution $M_{i}, i=1,2, \ldots, k$. Obtain $X_{s: n}$ under the 
candidate distribution $M_{i}$ and label it by $\widehat{X}_{s: n}^{M_{i}, j}$ for $s=r+$ $1, \ldots, n, i=1,2, \ldots, k$ and $j=1$ or 2 .

Step 3. Let $\widehat{X}_{s: n}^{A 1, j}$ denote the predicted value of $X_{s: n}$ for $j=$ 1 or 2 . Based on the method proposed by Dumonceaux and Antle [16], we can obtain $\widehat{X}_{s: n}^{A 1, j}$, which can provide the largest maximum likelihood information by

$$
\begin{gathered}
\widehat{L}_{A 1}\left(\widehat{\mu}_{A 1}, \widehat{\sigma}_{A 1}\right)=\max \left\{\widehat{L}_{M_{1}}\left(\widehat{\mu}_{M_{1}}, \widehat{\sigma}_{M_{1}}\right),\right. \\
\left.\widehat{L}_{M_{2}}\left(\widehat{\mu}_{M_{2}}, \widehat{\sigma}_{M_{2}}\right), \ldots, \widehat{L}_{M_{k}}\left(\widehat{\mu}_{M_{k}}, \widehat{\sigma}_{M_{k}}\right)\right\} .
\end{gathered}
$$

If the candidate distributions are ND and SEV, Steps 2 and 3 in Algorithm 1 can be reduced to Step 2' and Step 3' as the following, respectively:

Step 2'. Obtain $\left(\widehat{\mu}_{N}, \widehat{\sigma}_{N}\right),\left(\widehat{\mu}_{S}, \widehat{\sigma}_{S}\right), \widehat{L}_{N}\left(\widehat{\mu}_{N}, \widehat{\sigma}_{N}\right)$ and $\widehat{L}_{S}\left(\widehat{\mu}_{S}, \widehat{\sigma}_{S}\right)$. Obtain $X_{s: n}$ under the ND $\left(\widehat{X}_{s: n}^{N, j}\right)$ and obtain $X_{s: n}$ under the $\operatorname{SEV}\left(\widehat{X}_{s: n}^{S E V, j}\right)$ for $s=r+1, \ldots, n$ and $j=1$ or 2 .

Step 3'. Let $\widehat{X}_{s: n}^{A 1}$ denote the predicted value of $X_{s: n}$. Then

$$
\widehat{X}_{s: n}^{A 1}= \begin{cases}\widehat{X}_{s: n}^{N, j} & \text { if } \widehat{L}_{N}\left(\widehat{\mu}_{N}, \widehat{\sigma}_{N}\right)>\widehat{L}_{S}\left(\widehat{\mu}_{S}, \widehat{\sigma}_{S}\right) \\ \widehat{X}_{s: n}^{S E V, j}, & \text { otherwise. }\end{cases}
$$

$$
\text { for } s=r+1, \ldots, n \text { and } j=1 \text { or } 2 .
$$

Algorithm 2 (the $D_{S P}$ approach).

Step 1. Collect a type II censored sample, which has size $n$ and $r$ observed failure times.

Step 2. Obtain $\left(\widehat{\mu}_{M_{i}}, \widehat{\sigma}_{M_{i}}\right)$ for $i=1,2, \ldots, k$, and then obtain $\widehat{X}_{s: n}^{M_{i}, j}$ for $s=r+1, \ldots, n, i=1,2, \ldots, k$ and $j=1$ or 2 .

Step 3. Based on the method proposed by Castro-Kuriss et al. [29], the modification of $D_{S P}$ with censored observations can be presented by

$$
\begin{aligned}
& D_{S P}(\mu, \sigma) \\
& =\max _{1 \leq i \leq r}\left\{\frac{2}{\pi}\left|\arcsin \left(\sqrt{\frac{i-0.5}{n}}\right)-\arcsin \left(\sqrt{U_{i: n}}\right)\right|\right\},
\end{aligned}
$$

where $U_{i: n}=G\left(\left(x_{i: n}-\mu\right) / \sigma\right)$. The definition of $G(\bullet)$ is the same as that of (2), it represents the CDF of the assumed distribution in model selection. Evaluate the value of $D_{S P}$ through using the candidate distribution $M_{i}$ for $i=1,2, \ldots$, $k$.

Step 4. Let $\widehat{X}_{s: n}^{A 2, j}$ be the predicted value of $X_{s: n}$ for $j=1$ or 2 , then $\widehat{X}_{s: n}^{A 2, j}$ can be obtained with the smallest $\widehat{D}_{S P}$. That is, $\widehat{X}_{s: n}^{A 2, j}$ is the value corresponding to $\widehat{D}_{S P}^{A 2}\left(\widehat{\mu}_{A 2}, \widehat{\sigma}_{A 2}\right)$, which is defined by

$$
\begin{gathered}
\widehat{D}_{S P}^{A 2}\left(\widehat{\mu}_{A 2}, \widehat{\sigma}_{A 2}\right)=\min \left\{\widehat{D}_{S P}\left(\widehat{\mu}_{M_{1}}, \widehat{\sigma}_{M_{1}}\right),\right. \\
\left.\widehat{D}_{S P}\left(\widehat{\mu}_{M_{2}}, \widehat{\sigma}_{M_{2}}\right), \ldots, \widehat{D}_{S P}\left(\widehat{\mu}_{M_{k}}, \widehat{\sigma}_{M_{k}}\right)\right\} .
\end{gathered}
$$

If the candidate distributions are ND and SEV, Steps 2, 3, and 4 in Algorithm 2 can be reduced to Step 2' and Step 3' as the following, respectively:

Step 2'. Obtain $\left(\widehat{\mu}_{N}, \widehat{\sigma}_{N}\right)$ and $\left(\widehat{\mu}_{S}, \widehat{\sigma}_{S}\right)$. Obtain the $\widehat{X}_{s: n}^{N, j}$ under the ND and obtain the $\widehat{X}_{s: n}^{S E V, j}$ under the SEV for $s=r+1, \ldots, n$ and $j=1$ or 2 .

Step 3'. The modification of $D_{S P}$ with censored observations can be presented by

$$
\begin{aligned}
& D_{S P}(\mu, \sigma) \\
& =\max _{1 \leq i \leq r}\left\{\frac{2}{\pi}\left|\arcsin \left(\sqrt{\frac{i-0.5}{n}}\right)-\arcsin \left(\sqrt{U_{i: n}}\right)\right|\right\},
\end{aligned}
$$

where $U_{i: n}=G\left(\left(x_{i: n}-\mu\right) / \sigma\right)$. The definition of $G(\bullet)$ is the same as that of (2); it represents the CDF of the assumed distribution in model selection. Evaluate the values of $D_{S P}$ through using the ND and SEV and denot them by $\widehat{D}_{S P}^{N}\left(\widehat{\mu}_{N}, \widehat{\sigma}_{N}\right)$ and $\widehat{D}_{S P}^{S E V}\left(\widehat{\mu}_{S}, \widehat{\sigma}_{S}\right)$, respectively.

Step $4^{\text {'. Let }} \widehat{X}_{s: n}^{A 2, j}$ denote the predicted value of $X_{s: n}$, then $\widehat{X}_{s: n}^{A 2, j}$ can be obtained by

$$
\widehat{X}_{s: n}^{A 2}= \begin{cases}\widehat{X}_{s: n}^{N, j}, & \text { if } \widehat{D}_{S P}^{N}\left(\widehat{\mu}_{N}, \widehat{\sigma}_{N}\right)<\widehat{D}_{S P}^{S E V}\left(\widehat{\mu}_{S}, \widehat{\sigma}_{S}\right) \\ \widehat{X}_{s: n}^{S E V, j}, & \text { if } \widehat{D}_{S P}^{N}\left(\widehat{\mu}_{N}, \widehat{\sigma}_{N}\right) \geq \widehat{D}_{S P}^{S E V}\left(\widehat{\mu}_{S}, \widehat{\sigma}_{S}\right)\end{cases}
$$

for $s=r+1, \ldots, n$ and $j=1$ or 2 .

Algorithm 3 (the D approach).

Step 1. Collect a type II censored sample, which has size $n$ and $r$ observed failure times.

Step 2. Obtain $\left(\widehat{\mu}_{M_{i}}, \widehat{\sigma}_{M_{i}}\right)$ for $i=1,2, \ldots, k$, and then obtain $\widehat{X}_{s: n}^{M_{i}, j}$ for $s=r+1, \ldots, n, i=1,2, \ldots, k$ and $j=1$ or 2 .

Step 3. Based on the method proposed by Castro-Kuriss et al. [29], the modification of $D(\mu, \sigma)$ with censored observations can be presented by

$$
D(\mu, \sigma)=\max _{1 \leq i \leq r}\left\{\frac{2}{\pi}\left|\sqrt{\frac{i-0.5}{n}}-U_{i: n}\right|\right\}+\frac{0.5}{n},
$$

where $U_{i: n}=G\left(\left(x_{i: n}-\mu\right) / \sigma\right)$.

Step 4. Let $\widehat{X}_{s: n}^{A 3, j}$ be the predicted value of $X_{s: n}$ for $j=1$ or 2 , then $\widehat{X}_{s: n}^{A 3, j}$ can be obtained with the smallest $\widehat{D}\left(\widehat{\mu}_{M_{i}}, \widehat{\sigma}_{M_{i}}\right)$. That is, $\widehat{X}_{s: n}^{A 3, j}$ is the value corresponding to $\widehat{D}^{A 3}\left(\widehat{\mu}_{A 3}, \widehat{\sigma}_{A 3}\right)$, which is defined by

$$
\begin{aligned}
& \widehat{D}^{A 3}\left(\widehat{\mu}_{A 3}, \widehat{\sigma}_{A 3}\right)=\min \left\{\widehat{D}\left(\widehat{\mu}_{M_{1}}, \widehat{\sigma}_{M_{1}}\right), \widehat{D}\left(\widehat{\mu}_{M_{2}}, \widehat{\sigma}_{M_{2}}\right),\right. \\
& \left.\ldots, \widehat{D}\left(\widehat{\mu}_{M_{k}}, \widehat{\sigma}_{M_{k}}\right)\right\} .
\end{aligned}
$$


If the candidate distributions are ND and SEV, Steps 2, 3, and 4 in Algorithm 3 can be reduced to Step 2' and Step 3' as the following, respectively:

Step 2'. Obtain $\left(\widehat{\mu}_{N}, \widehat{\sigma}_{N}\right)$ and $\left(\widehat{\mu}_{S}, \widehat{\sigma}_{S}\right)$. Obtain $\widehat{X}_{s: n}^{N, j}$ under the ND and obtain $\widehat{X}_{s: n}^{S E V, j}$ under the SEV for $s=r+1, \ldots, n$ and $j=1$ or 2 .

Step 3'. The modification of $D(\mu, \sigma)$ with censored observations can be presented by

$$
D(\mu, \sigma)=\max _{1 \leq i \leq r}\left\{\frac{2}{\pi}\left|\sqrt{\frac{i-0.5}{n}}-U_{i: n}\right|\right\}+\frac{0.5}{n},
$$

where $U_{i: n}=G\left(\left(x_{i: n}-\mu\right) / \sigma\right)$. Evaluate the value of $D(\mu, \sigma)$ by using the ND and SEV and denote them by $\widehat{D}^{N}\left(\widehat{\mu}_{N}, \widehat{\sigma}_{N}\right)$ and $\widehat{D}^{S E V}\left(\widehat{\mu}_{S}, \widehat{\sigma}_{S}\right)$.

Step $4^{\prime}$. Let $\widehat{X}_{s: n}^{A 3, j}$ denote the predicted value of $X_{s: n}$, then $\widehat{X}_{s: n}^{A 3, j}$ can be obtained by

$$
\begin{array}{r}
\widehat{X}_{s: n}^{A 3, j}= \begin{cases}\widehat{X}_{s: n}^{N, j}, & \text { if } \widehat{D}^{N}\left(\widehat{\mu}_{N}, \widehat{\sigma}_{N}\right)<\widehat{D}^{S E V}\left(\widehat{\mu}_{S}, \widehat{\sigma}_{S}\right) \\
\widehat{X}_{s: n}^{S E V, j}, & \text { if } \widehat{D}^{N}\left(\widehat{\mu}_{N}, \widehat{\sigma}_{N}\right) \geq \widehat{D}^{S E V}\left(\widehat{\mu}_{S}, \widehat{\sigma}_{S}\right)\end{cases} \\
\text { for } s=r+1, \ldots, n \text { and } j=1 \text { or } 2 .
\end{array}
$$

\section{Monte Carlo Simulations}

A Monte Carlo simulation study was conducted in this section, by using $\mathrm{R}$ language, to evaluate the performance of the proposed three approaches with two predicting methods. We consider the ND and SEV as the candidate distributions for competing the best lifetime model in the simulation study. The data sets of type II censoring sample, $x_{1: n}, \ldots, x_{r: n}$, used in the simulation were randomly generated from the ND and SEV with location parameter $\mu=0$ and scale parameter $\sigma=$ 1 . Then, the $s^{\text {th }}$ order statistic is predicted and denoted by $\widehat{X}_{s: n}$ for $s=r+1, r+2, \ldots, n$ for the sample sizes $n=20,30,40,50$ and 60 . For the purpose of comparison, the values of the bias and mean square error (MSE) of $\widehat{X}_{s: n}$ are evaluated using $N=10000$ Monte Carlo runs:

$$
\text { bais }=\frac{1}{N} \sum_{i=1}^{N}\left(\widehat{X}_{s: n, i}-X_{s: n}\right)
$$

and

$$
\operatorname{MSE}=\frac{1}{N} \sum_{i=1}^{N}\left(\widehat{X}_{s: n, i}-X_{s: n}\right)^{2}
$$

where $\widehat{X}_{s: n, i}$ is the predicted value of $X_{s: n}$ that is obtained in the $i^{\text {th }}$ iteration of simulation for $i=1, \ldots, N$. All simulation results are displayed in Tables 1 and 2 with the candidate distributions of ND and SEV. From Tables 1 and 2, we notice that the bias and MSE are large when the misspecification model is used. The impact of misspecification depends on the values of $r$ and $s$. As $n$ or $r$ increases, the simulated bias and MSE are decreased. We also find that the MSE based on using the Taylor series prediction method is smaller than that based on using the expected values prediction method when the sample size is or larger than 30 .

To evaluate the performance of the three proposed model selection approaches for MLP, Tables 3-5 report the simulation results for three model selection approaches from the ND. Tables 6-8 respectively report the simulation results for three model selection approaches from the SEV. The column "correct (\%)" presented in Tables 3-8 is the correct model selection rate in all simulation runs. From Tables 3-8 we find that the three model selection approaches have good ability to identify the correct underlying distribution with a high probability. Moreover, the MSEs of these three approaches are close to those simulated MSEs of the cases by using the real underlying distribution. Overall, the correct model selection rates through using $D_{S P}$ approach or $D$ approach are higher than that of using the RRML approach when the sample size is smaller than 30. When the sample size grows to or over 30 , the performance of the RRML approach is improved and the correct model selection rate of the RRML approach is higher than that are obtained by using the $D_{S P}$ or $D$ approach. To compare the performance of using two different MLPs, the MSEs of using the expected values prediction method are smaller than that using the Taylor series prediction method when the sample size is smaller than 30 . The proposed approaches can perform well under large sample size cases.

\section{Illustrative Examples}

In this section, three numerical examples are presented to illustrate the proposed approaches in Sections 2-4.

5.1. Example 1. A test airplane component's failure time dataset provided in Mann and Fertig [30], in which 13 components were placed on test, and the test was terminated at the time of the $10^{\text {th }}$ failure. The failure times (in hours) of the 10 components that failed were

$D_{1}: 0.22,0.50,0.88,1.00,1.32,1.33,1.54,1.76,2.50$, 3.00 .

Let $Y_{1}$ be the logs of the ten observations, i.e., $Y_{1}=\ln \left(D_{1}\right)$. Figure 2 presents the histogram and the estimated PDFs of the ND and SEV. From Figure 2, we find a difficulty to fully decide the best distribution for lifetime fitting due to the fact that both candidate distributions can provide good fitting for this data set. In this example, we consider using $D_{S P}$ approach to discriminate competing models and apply Taylor series prediction method to predicting the future order statistics, which are censored. The $\mathrm{R}$ source codes of Example 1 can be found in Appendix $\mathrm{C}$ and other designs can be obtained from the authors upon request. 


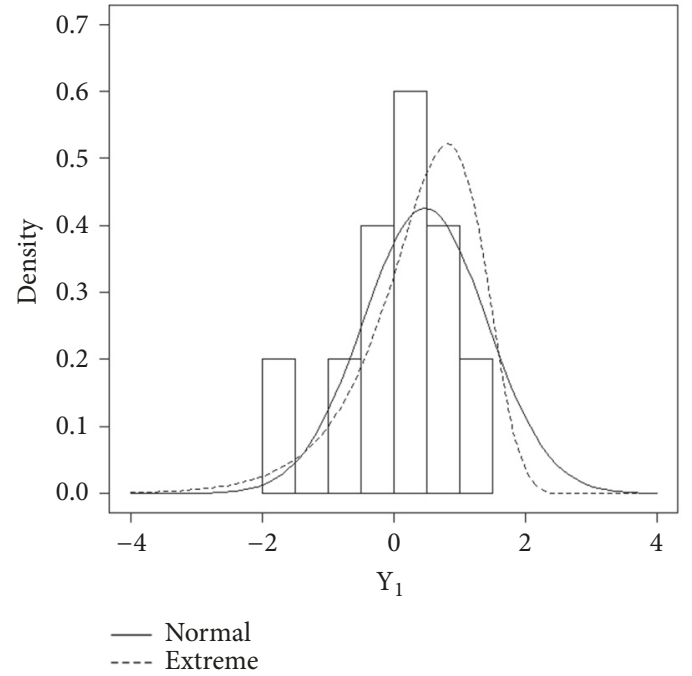

FIGURE 2: The histogram and the estimated probability density functions of airplane component's failure time in Example 1.

Through using Newton-Raphson algorithm, we obtained the MLEs of $\mu$ and $\sigma$ as $\left(\widehat{\mu}_{N}, \widehat{\sigma}_{N}\right)=(0.479,0.938)$ and $\left(\widehat{\mu}_{S}, \widehat{\sigma}_{S}\right)=(0.821,0.705)$ for the ND and SEV, respectively.

The $D_{S P}$ values via using ND and SEV are 0.223 and 0.212 , respectively. Because the $D_{S P}$ value obtained from the SEV is smaller than that obtained from the ND, we claim the best distribution of this data set is SEV. The Taylor series prediction for $\left(Y_{11: 13}, Y_{12: 13}, Y_{13: 13}\right)$ under the extreme value distribution with the censored sample can be obtained by $\left(\widehat{Y}_{11: 13}^{A 2,2}, \widehat{Y}_{12: 13}^{A 2,2}, \widehat{Y}_{13: 13}^{A 2,2}\right)=(1.098,1.281,1.567)$.

5.2. Example 2. In this example, we consider that the tests on endurance of deep groove ball bearings data, reported by Lieblein and Zelen [31] and further studied by Meeker and Escobar (1998), are used to illustrate the methodologies developed in this paper. The data are the numbers of million revolutions before failure for each of the 23 ball bearings in the life test. Meeker and Escobar [32] pointed out that this data $\left(D_{2}\right)$ follows lognormal distribution or Weibull distribution. Hence $Y_{2}=\ln \left(D_{2}\right)$ follows a ND or SEV. The data is given as follows:

$D_{2}: 17.88,28.92,33.00,41.52,42.12,45.60,48.40,51.84$, 51.96, 54.12, 55.56, 67.80, 68.64, 68.64, 68.88, 84.12, $93.12,98.64,105.12,105.84,127.92,128.04,173.40$.

For more information about this carbon fiber breaking strength data set, one can be referred to Meeker and Escobar (1998). In this example, we assume that the censoring proportion is $0.8696(r=20, n=23)$. Figure 3 presents the histogram and the estimated PDFs of ND and SEV based on the type II right-censored data set. From Figure 3, it is difficult to decide the best distribution from these two candidate distributions.

We consider using $D$ approach in Example 2 for model selection and use expected values prediction method to

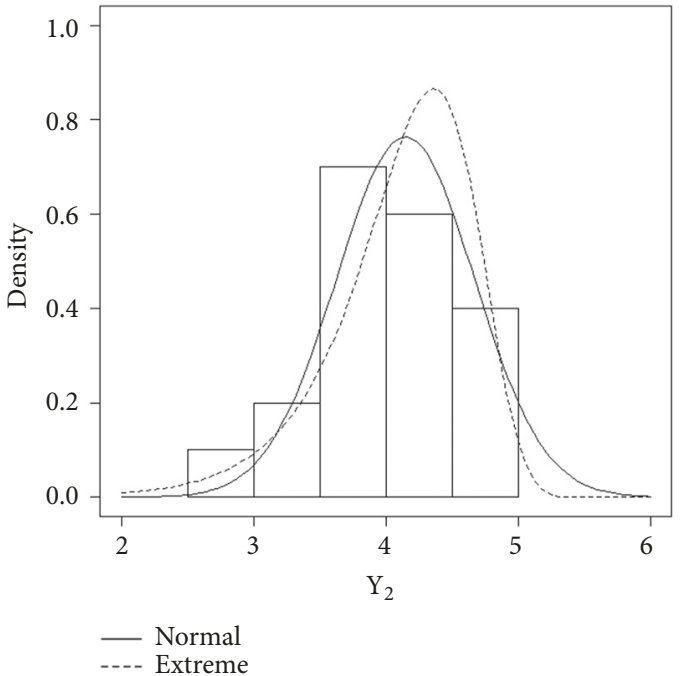

FIGURE 3: The histogram and the estimated probability density functions of tests on endurance of deep groove ball bearings in Example 2.

predict the future order statistics, which are censored. The MLEs of $\mu$ and $\sigma$ can be obtained via using Newton-Raphson algorithm, the resulting MLEs are $\left(\widehat{\mu}_{N}, \widehat{\sigma}_{N}\right)=(4.148,0.524)$ and $\left(\widehat{\mu}_{S}, \widehat{\sigma}_{S}\right)=(4.369,0.425)$ for the ND and SEV, respectively. The $D$ values based on using the ND and SEV are 0.181 and 0.297, respectively. Because the $D$ value obtained from ND is smaller than that obtained from SEV, we claim the best model is normal. The expected values prediction of $\left(Y_{21: 23}, Y_{22: 23}, Y_{23: 23}\right)$ via using ND are $\left(\widehat{Y}_{21: 23}^{A 3,1}, \widehat{Y}_{22: 23}^{A 3,1}, \widehat{Y}_{23: 23}^{A 3,1}\right)=$ $(4.784,4.922,5.160)$. In addition, we compare our prediction results with the MMLP values that proposed by Yang and Tong (2006), in which the MMLP is $\left(\widehat{Y}_{21: 23}, \widehat{Y}_{22: 23}, \widehat{Y}_{23: 23}\right)=$ $(4.662,4.936,5.175)$. Our predicted results are close to that proposed by Yang and Tong [14] even we cannot initially assume which one of the ND or SEV is the best distribution.

5.3. Example 3. We consider the experiment on the pulloff performance for use in automotive engine components, reported by Byrne and Taguchi [33] and further studied by Yang and Tong [14], is used to illustrate the methodologies developed in this study. An experiment was conducted to find a method to maximize the pull-off force. Four control factors that could influence the assembly's pull-off force have been identified. Repeat 8 times for each run and record the pull-off force in pounds. Table 9 lists the four control factors with their levels and complete data of this experiment. In this example, we assume that the censoring proportion is 0.75 $(r=6, n=8)$. Please note that censored data cannot support the practitioner to conduct experimental design methods. Predicting the unobserved data and using a pseudo-complete data set for conducting experimental design methods is required.

We consider using the RRML approach for model selection and use Taylor series prediction method to predict 


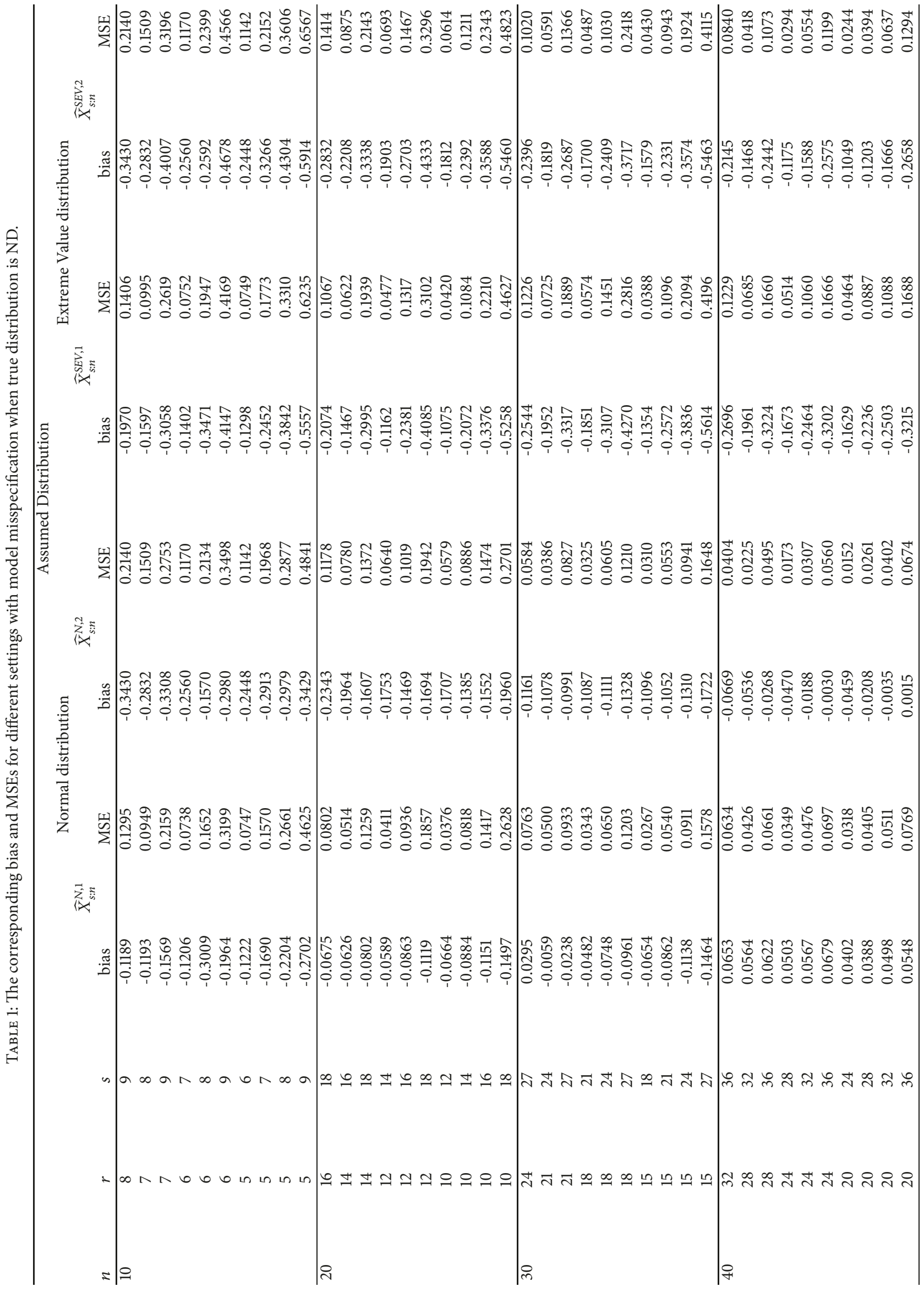




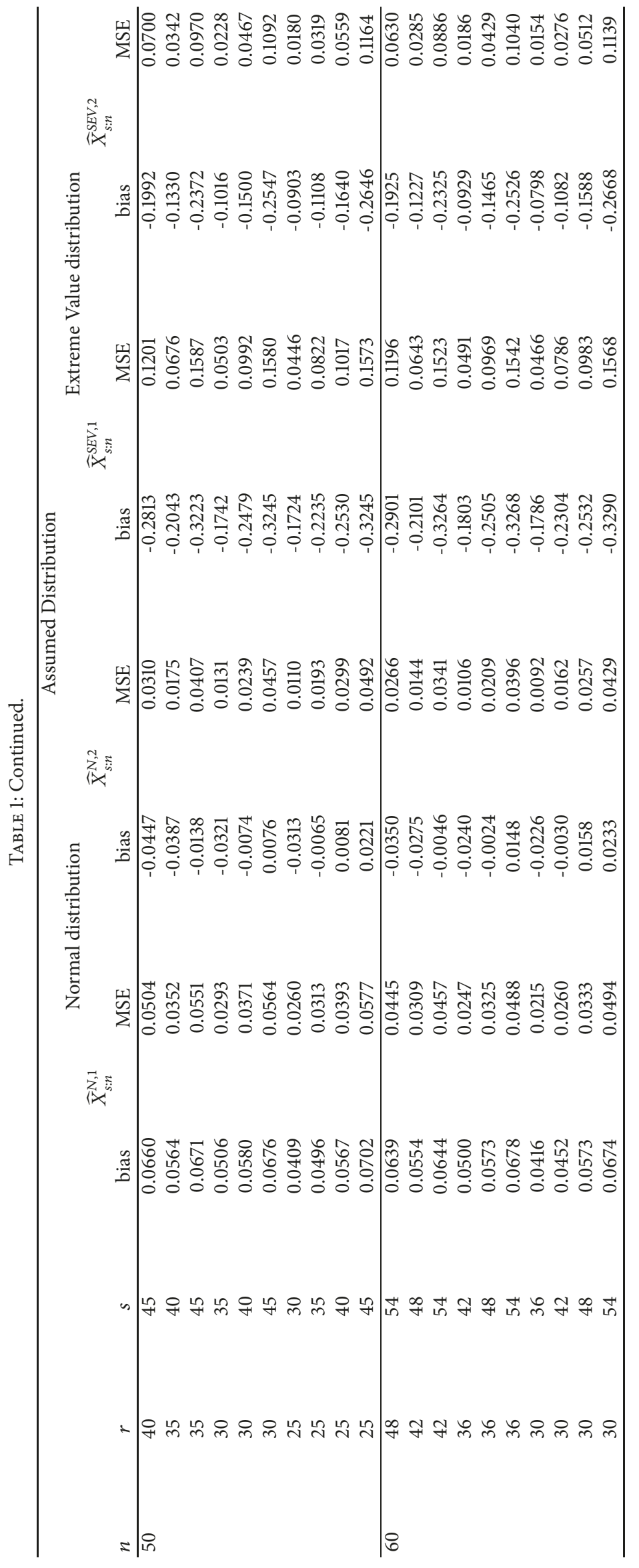




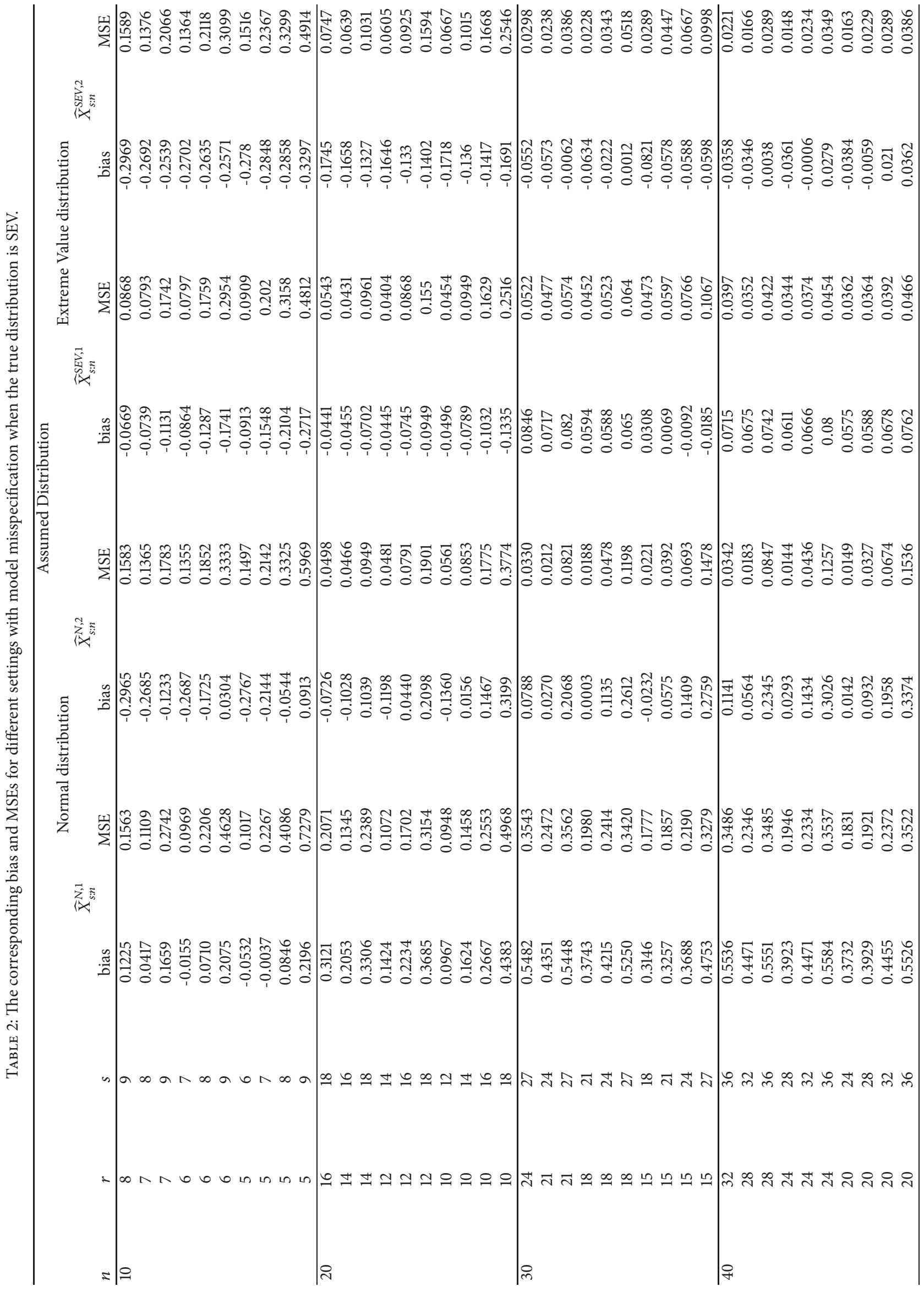




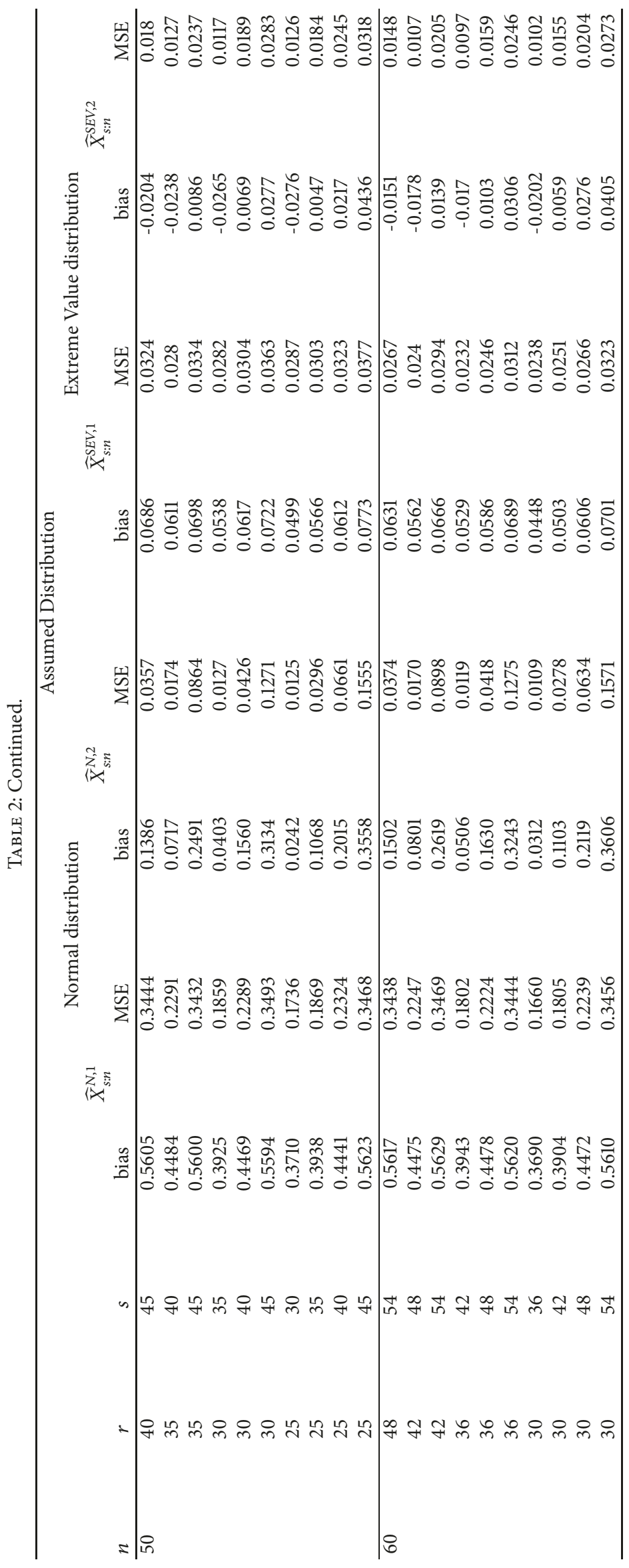




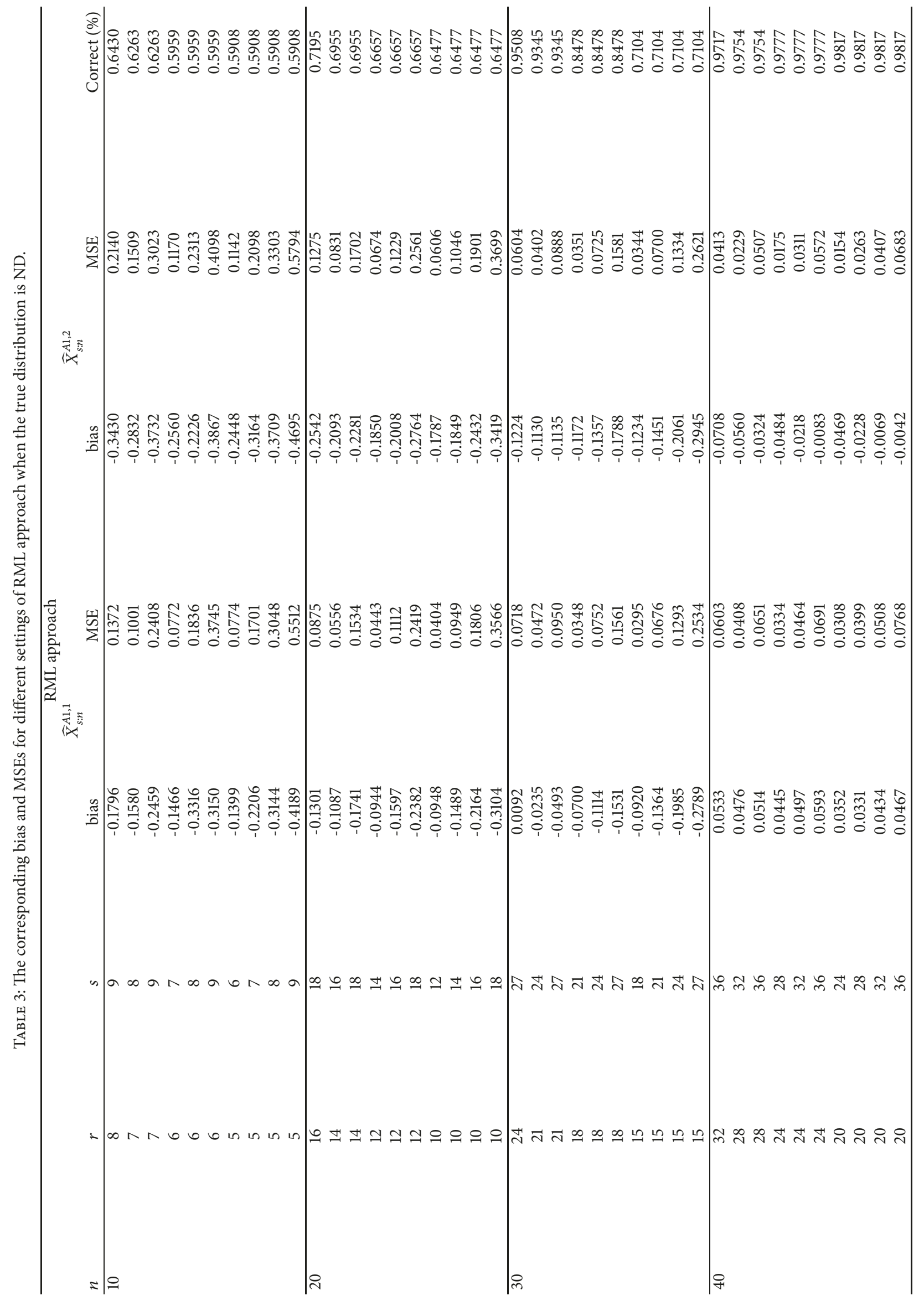




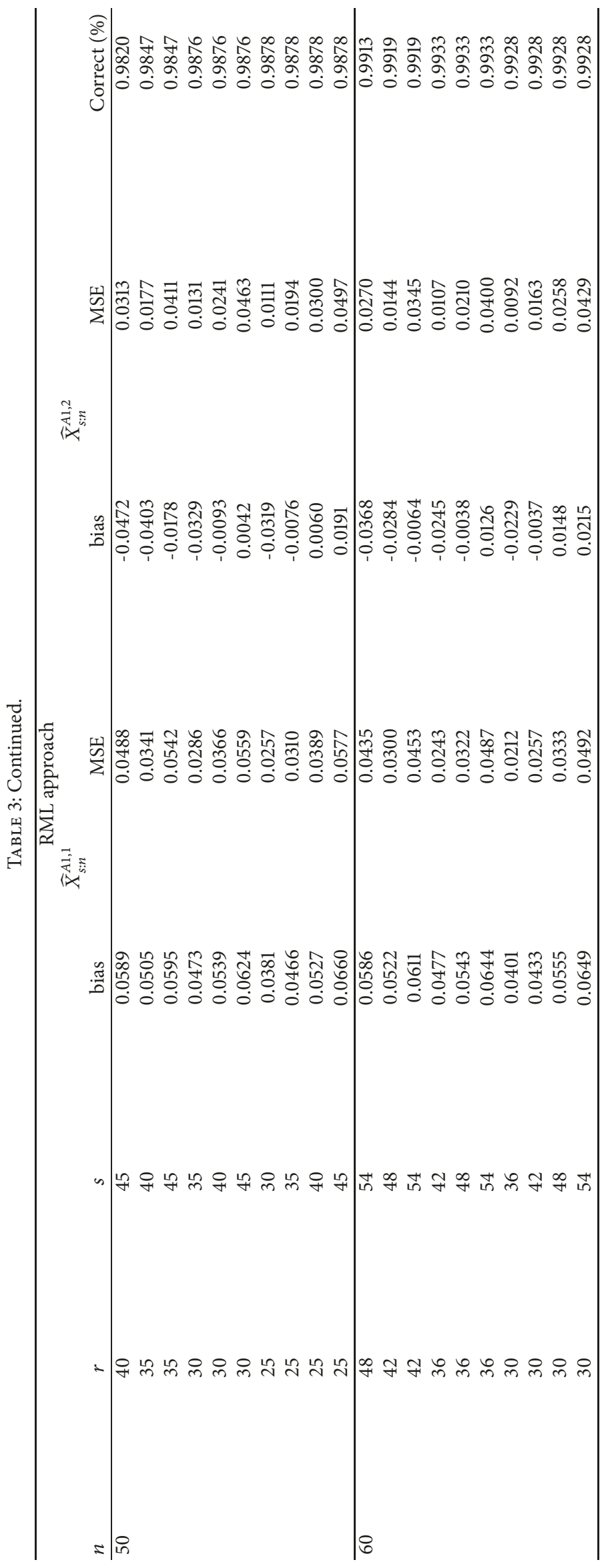




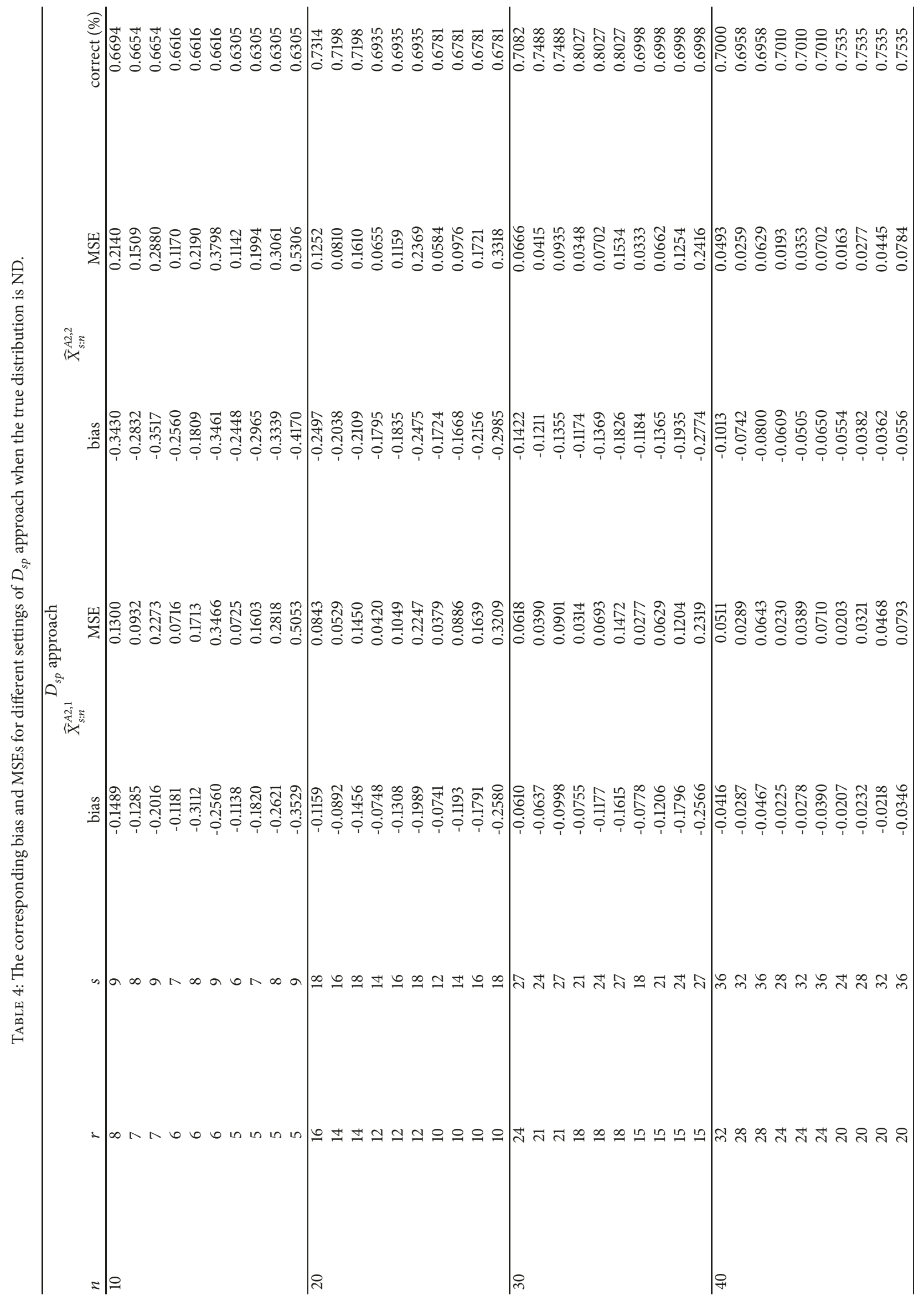




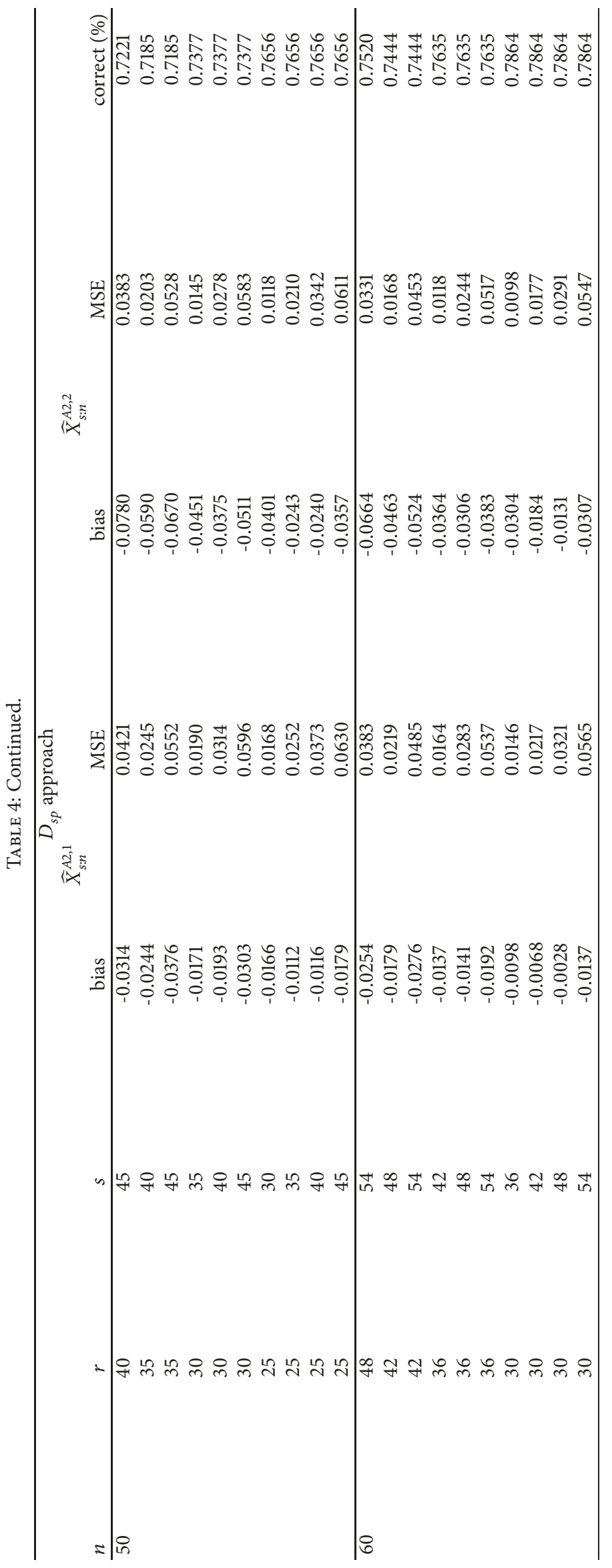




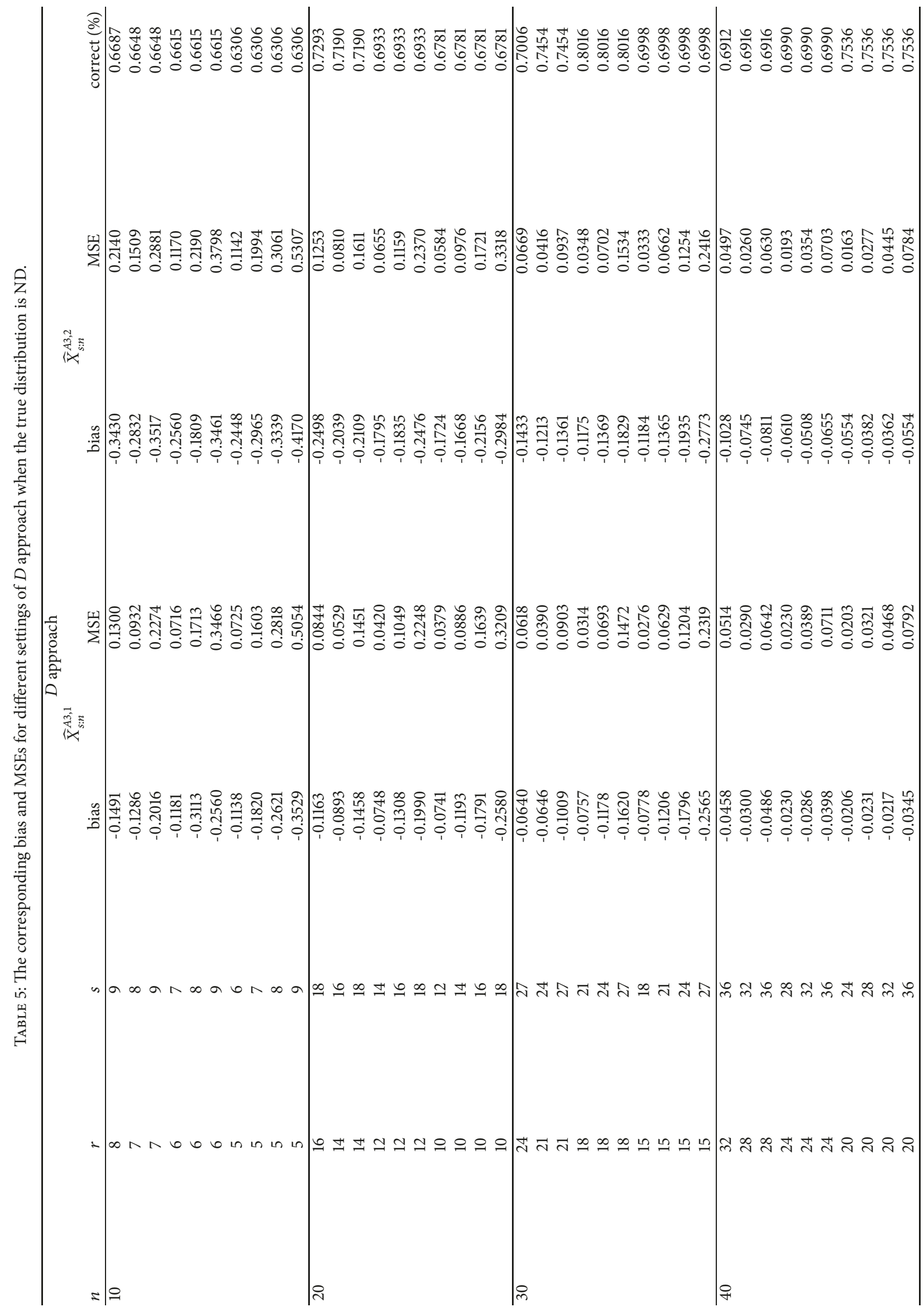




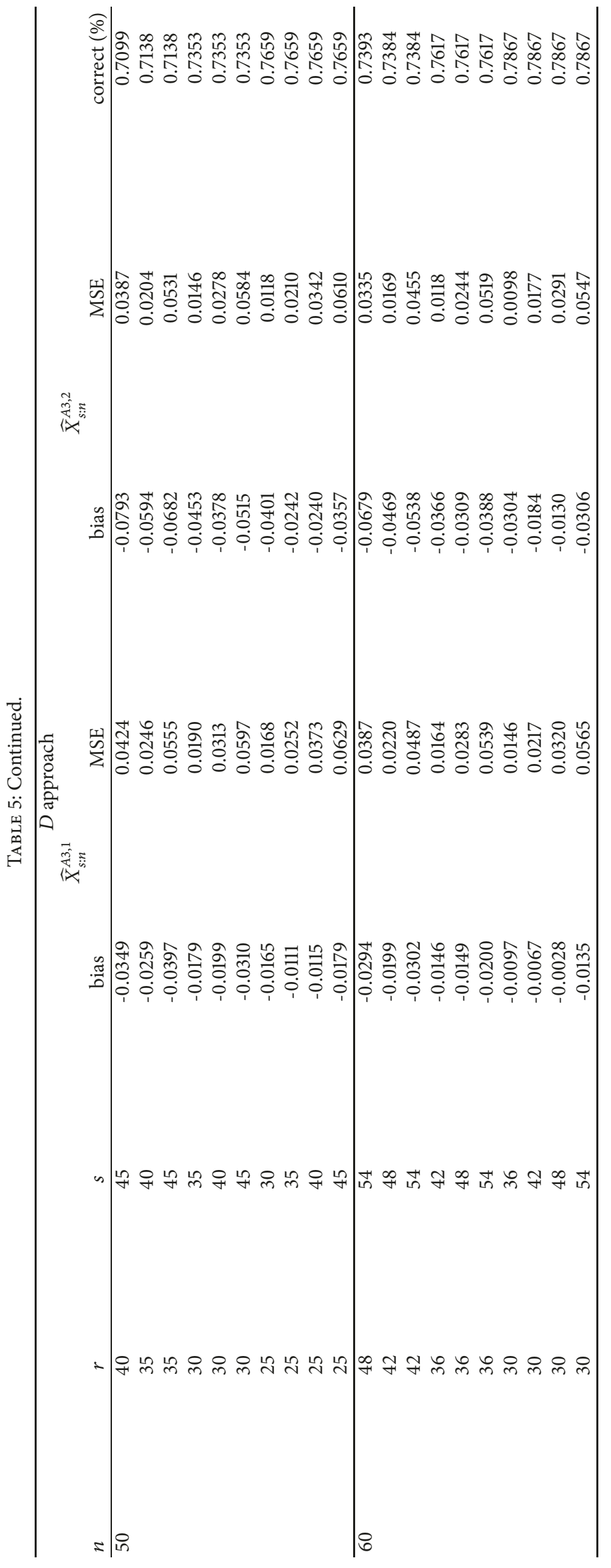




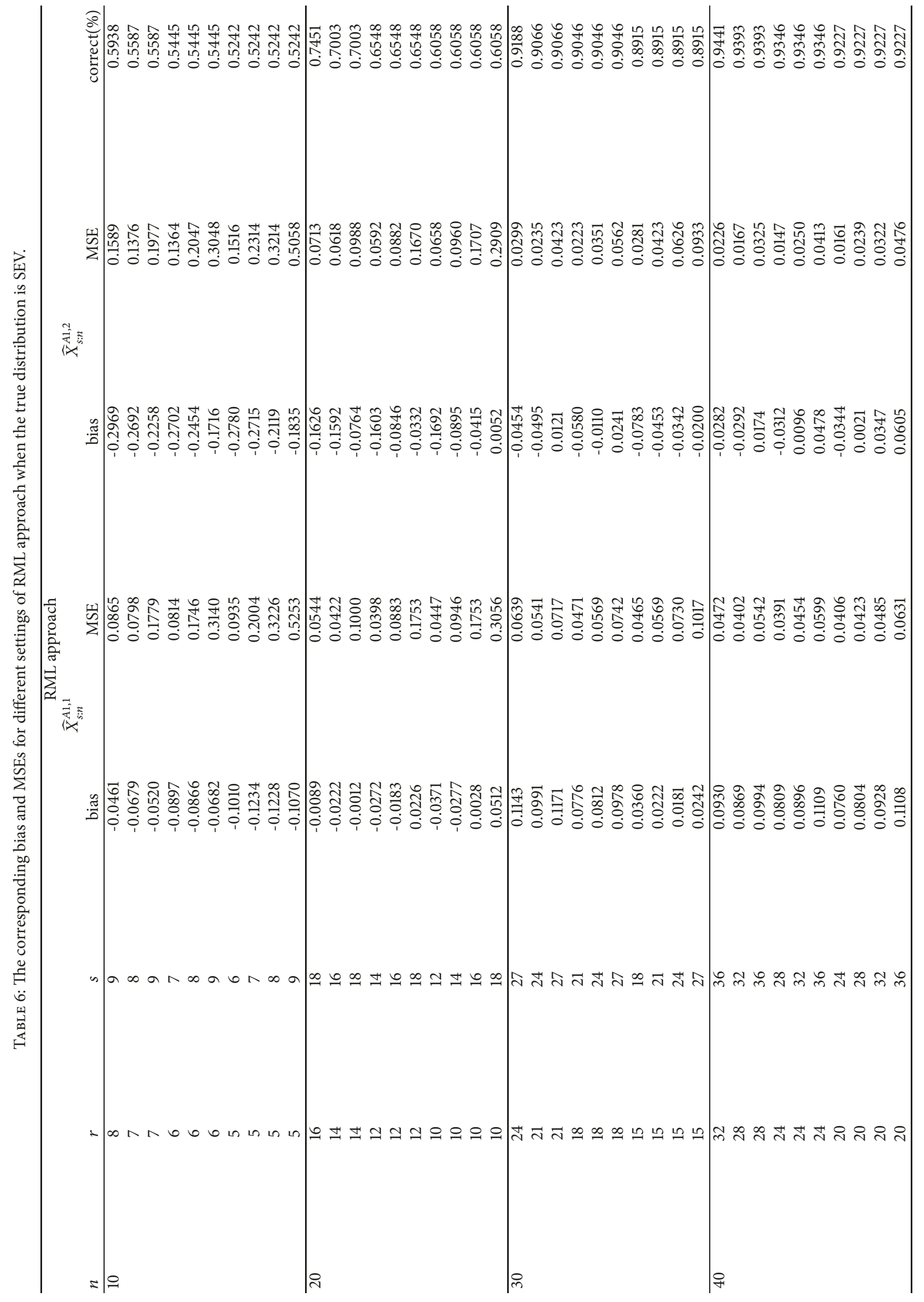




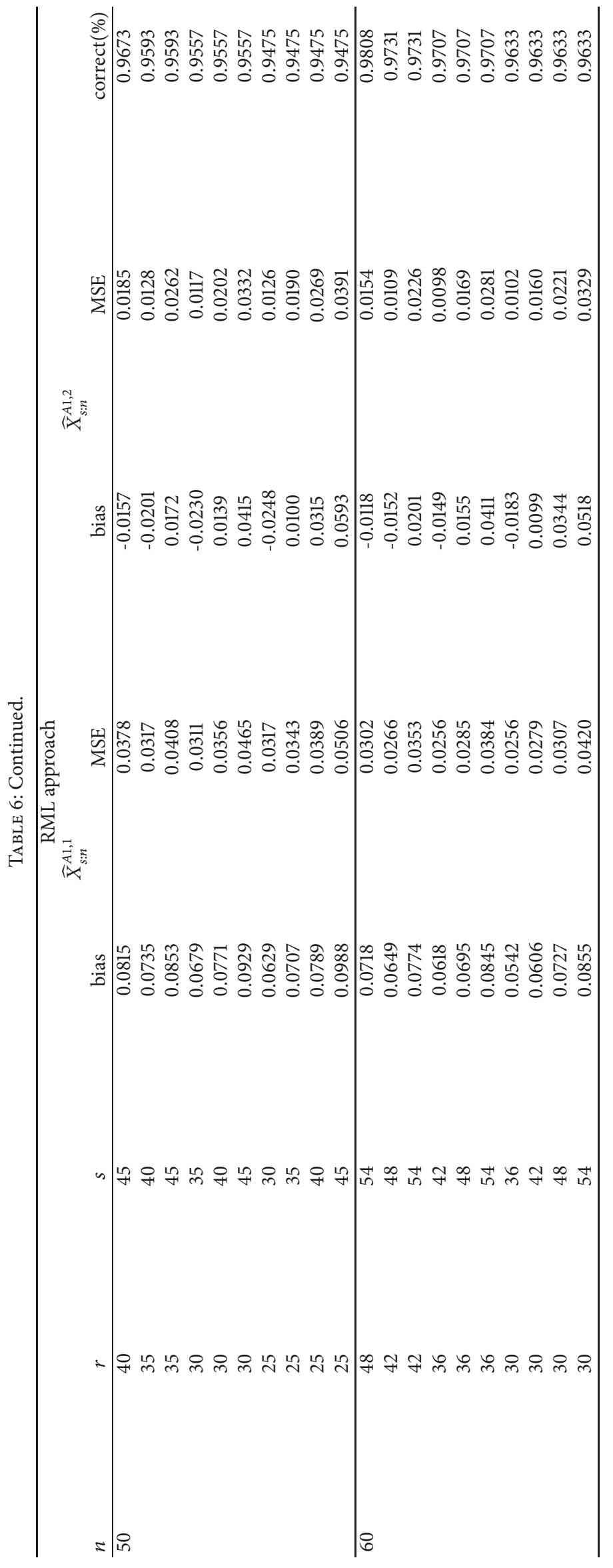




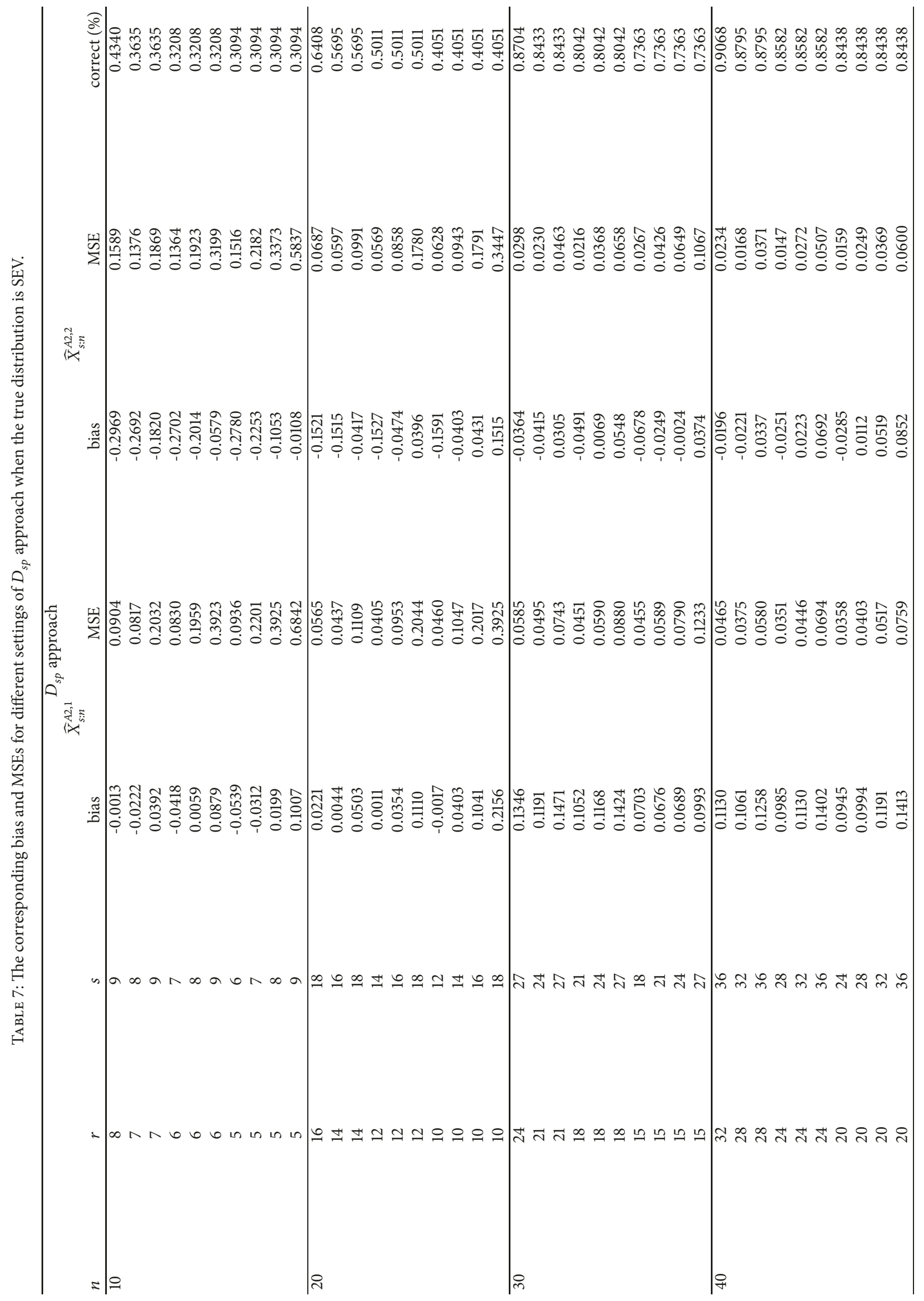




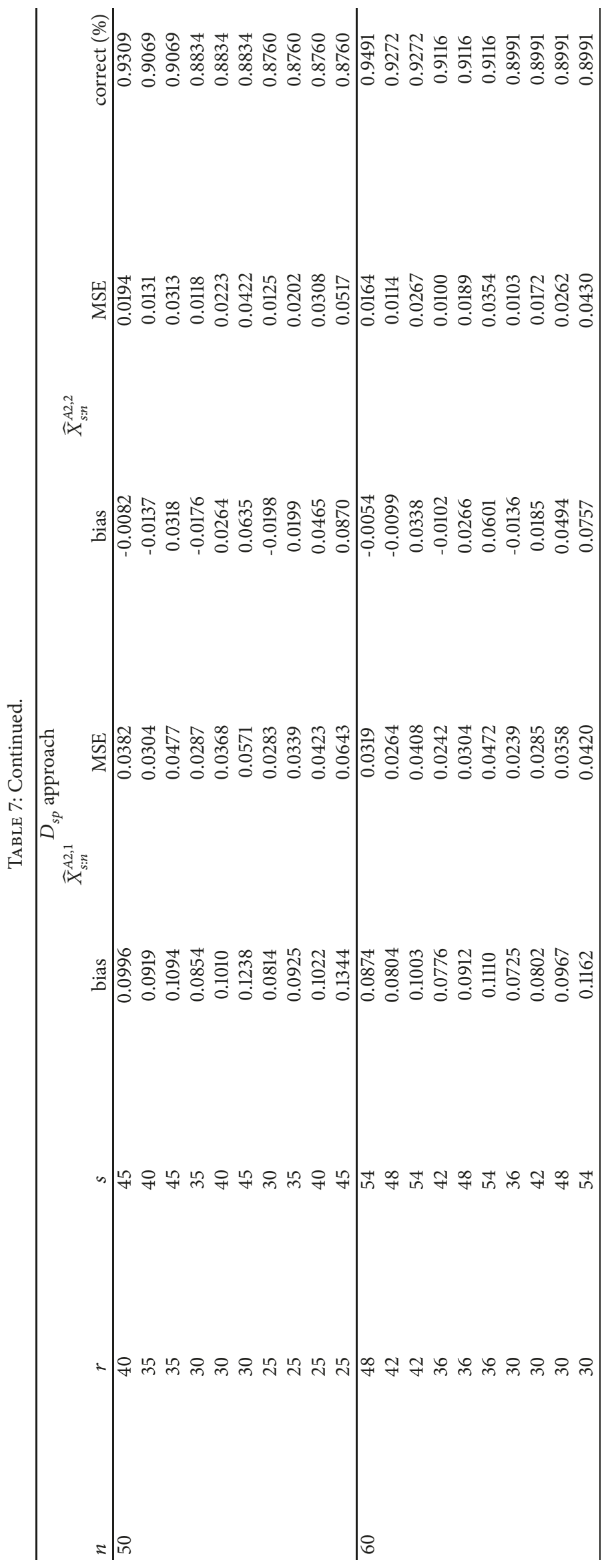




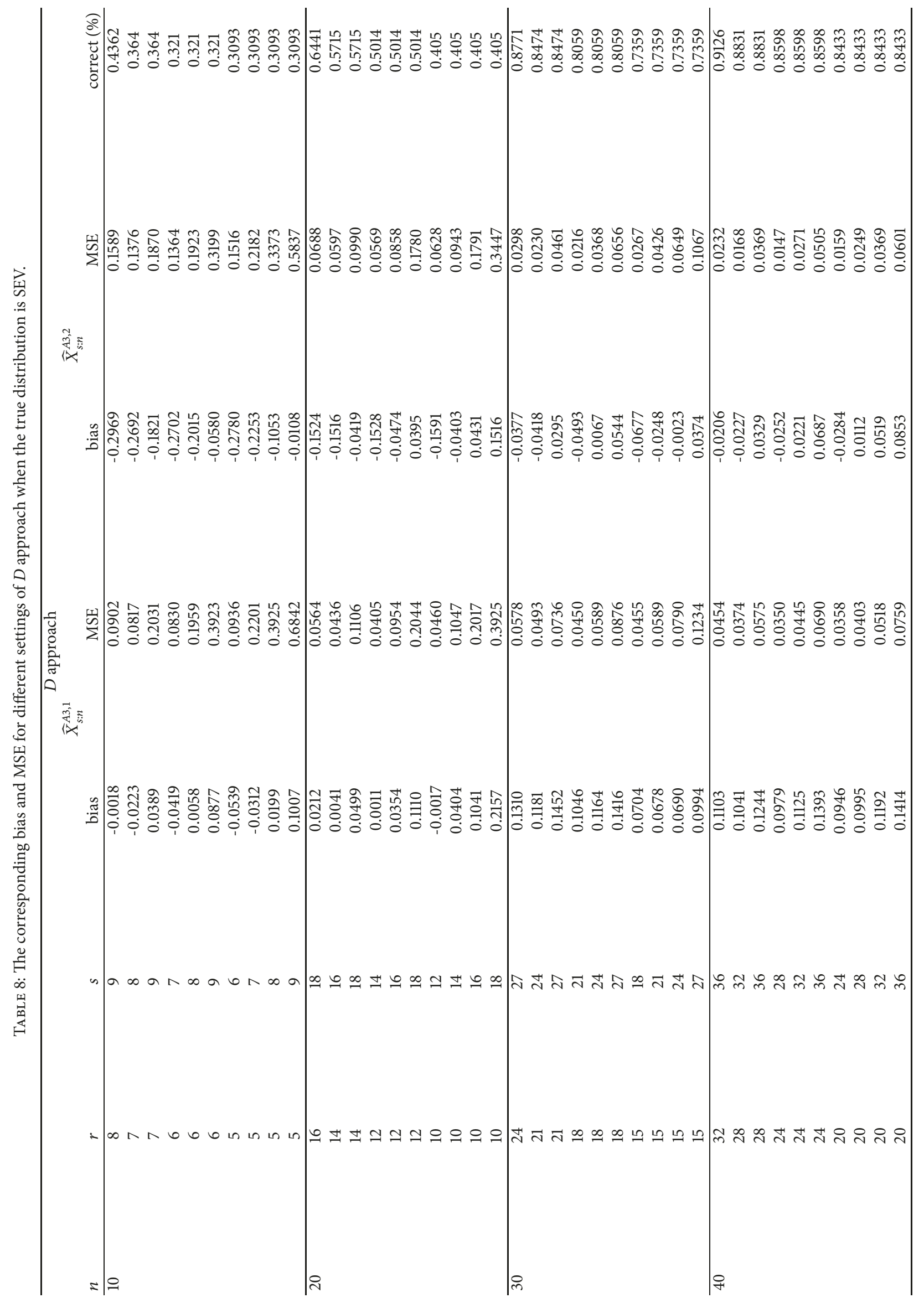




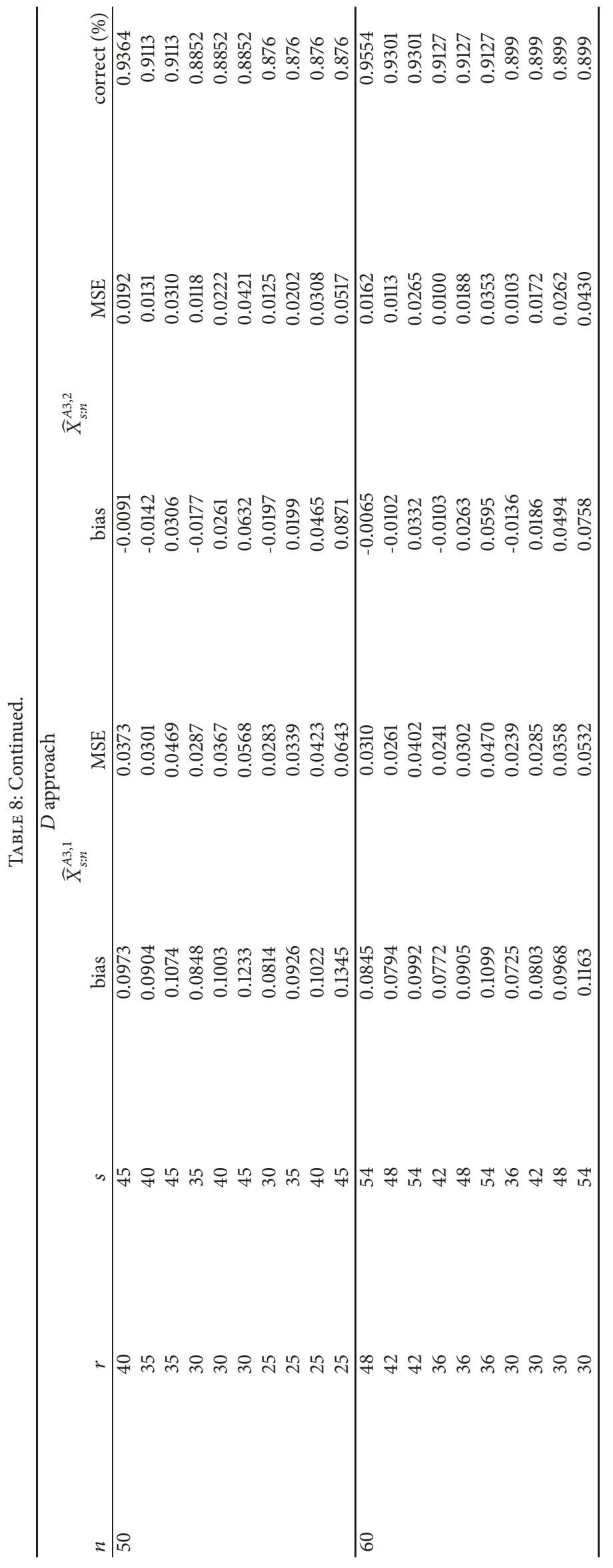


TABLE 9: Factors with levels of each factor and complete data in the experiments.

\begin{tabular}{lcccccccccccc}
\hline \multirow{2}{*}{ Run } & \multicolumn{3}{c}{ Factor } & \multicolumn{1}{c}{ Pull-off force for replicate } \\
& $\mathrm{A}$ & $\mathrm{B}$ & $\mathrm{C}$ & $\mathrm{D}$ & $x_{1: 8}$ & $x_{2: 8}$ & $x_{3: 8}$ & $x_{4: 8}$ & $x_{5: 8}$ & $x_{6: 8}$ & $x_{7: 8}$ & $x_{8: 8}$ \\
\hline 1 & 1 & 1 & 1 & 1 & 9.5 & 15.6 & 16.9 & 19.1 & 19.6 & 19.6 & 19.9 & 20 \\
2 & 1 & 2 & 2 & 2 & 15 & 16.2 & 19.4 & 19.6 & 19.7 & 19.8 & 21.9 & 24.2 \\
3 & 1 & 3 & 3 & 3 & 15.6 & 16.3 & 16.7 & 18.2 & 19.1 & 20.4 & 22.6 & 23.3 \\
4 & 2 & 1 & 2 & 3 & 17.4 & 18.3 & 18.6 & 18.9 & 18.9 & 21 & 23.2 & 24.7 \\
5 & 2 & 2 & 3 & 1 & 18.6 & 19.4 & 19.7 & 21.4 & 25.1 & 25.3 & 25.6 & 27.5 \\
6 & 2 & 3 & 1 & 2 & 14.7 & 16.2 & 16.3 & 19.6 & 19.8 & 20 & 22.5 & 24.7 \\
7 & 3 & 1 & 3 & 2 & 16.4 & 16.8 & 18.4 & 18.6 & 19.1 & 21.6 & 23.6 & 24.3 \\
8 & 3 & 2 & 1 & 3 & 14.2 & 15.1 & 15.6 & 16.8 & 17.8 & 19.6 & 23.2 & 24.4 \\
9 & 3 & 3 & 2 & 1 & 16.1 & 17.3 & 19.3 & 19.9 & 22.6 & 22.7 & 23.1 \\
\hline
\end{tabular}

Note: Factor A is interference with Low (1), Medium (2) and High (3) levels.

Factor B is connector wall thickness with Thin (1), Medium (2) and Thick (3) levels.

Factor C is insertion depth with Shallow (1), Medium (2) and Deep (3) levels.

Factor D is Percent adhesive in connector pre-dip with Low (1), Medium (2) and High (3) levels.

TABLE 10: The pseudo-complete data and results of model selection.

\begin{tabular}{|c|c|c|c|c|c|c|c|c|c|}
\hline \multirow{2}{*}{ Run } & \multicolumn{8}{|c|}{ Pull-off force for replicate } & \multirow{2}{*}{ Model selection } \\
\hline & $x_{1: 8}$ & $x_{2: 8}$ & $x_{3: 8}$ & $x_{4: 8}$ & $x_{5: 8}$ & $x_{6: 8}$ & $\widehat{X}_{7: 8}^{A 1,2}$ & $\widehat{X}_{8: 8}^{A 1,2}$ & \\
\hline 1 & 9.5 & 15.6 & 16.9 & 19.1 & 19.6 & 19.6 & 19.6 & 21.2 & SEV \\
\hline 2 & 15 & 16.2 & 19.4 & 19.6 & 19.7 & 19.8 & 19.8 & 20.73 & SEV \\
\hline 3 & 15.6 & 16.3 & 16.7 & 18.2 & 19.1 & 20.4 & 20.4 & 21.6 & ND \\
\hline 4 & 17.4 & 18.3 & 18.6 & 18.9 & 18.9 & 21 & 21 & 21.8 & ND \\
\hline 5 & 18.6 & 19.4 & 19.7 & 21.4 & 25.1 & 25.3 & 25.3 & 27.3 & ND \\
\hline 6 & 14.7 & 16.2 & 16.3 & 19.6 & 19.8 & 20 & 20 & 21.1 & SEV \\
\hline 7 & 16.4 & 16.8 & 18.4 & 18.6 & 19.1 & 21.6 & 21.6 & 22.8 & ND \\
\hline 8 & 14.2 & 15.1 & 15.6 & 16.8 & 17.8 & 19.6 & 19.6 & 20.9 & ND \\
\hline 9 & 16.1 & 17.3 & 19.3 & 19.9 & 22.6 & 22.7 & 22.7 & 24.5 & $\mathrm{ND}$ \\
\hline
\end{tabular}

the future order statistics in this example. After combining the uncensored data and the predicted censored data, the pseudo-complete data are shown in Table 10.

\section{Conclusions}

It could be difficult to discriminate a best model sometimes from several candidate distributions. The sample size, estimation methods, and goodness-of-fit testing methods can affect the final results of model selection. In this study, we focus on providing reliable methods to obtain predicting values of censored data to reduce the impact of model misspecification. In this study, three model selection approaches are proposed for predicting the future order statistics from type II censored data, in which the quality characteristic is assumed to follow a location-scale family. The ND and SEV are considered as the candidate members in the location-scale distribution to compete the best underlying distribution. The ND can be the log transformation from the lognormal distribution and the SEV can be the log transformation from the Weibull distribution. Discrimination between lognormal and Weibull distributions is equivalent to the discrimination between ND and SEV. Hence, both ND and SEV are widely used for practical reliability applications.

Through any one of three proposed approaches, the robust predictions can be obtained even under model uncertainty. Three examples are used to illustrate the methodologies. Moreover, the performance of these three proposed approaches are evaluated through using Monte Carlo simulations. Numerical results show that the three proposed model selection approaches are robust and effective in obtaining good predicted values for the future order statistics, which are censored.

In comparing these three proposed approaches, we recommend using $D_{S P}$ approach or $D$ approach for model selection and use expected values prediction method to predict the future order statistics for small sample size cases, that is, the sample cases with a size $n$ is less than 30. For large sample size cases (sample size $n$ larger than 30 ), we recommend using RRML approach for model selection and use Taylor series prediction method to predict the future order statistics. Simulation results show that the proposed approaches are robust and can highly reduce the impact caused by model uncertainty. The proposed approaches can 
also work well if more than two candidate distribution are competing for the best distribution.

Other model selection methods from the current three proposed approaches could also be competitive. How to employ new model selection methods for the topic of type II censored data prediction can be studied in the future.

\section{Appendix}

A.

For the normal distribution case, the functions $h\left(Z_{s: n}\right)$ and $h_{1}\left(z_{r: n}, Z_{s: n}\right)$ can be expanded by using Taylor series at the points $F^{-1}\left(p_{s}\right)$ and $\left(F^{-1}\left(p_{r}\right), F^{-1}\left(p_{s}\right)\right)$, respectively. We obtain

$$
h\left(z_{s: n}\right)=\frac{f\left(Z_{s: n}\right)}{1-F\left(Z_{s: n}\right)} \approx \alpha+\beta Z_{s: n}
$$

and

$$
\begin{aligned}
h_{1}\left(z_{r: n}, Z_{s: n}\right) & =\frac{f\left(Z_{s: n}\right)}{F\left(Z_{s: n}\right)-F\left(z_{r: n}\right)} \\
& \approx \gamma+\rho z_{r: n}-v_{s} Z_{s: n}
\end{aligned}
$$

in which the constants can be taken to be

$$
\begin{aligned}
& \alpha=\frac{f\left(\eta_{s}\right)\left\{\left(1+\eta_{s}^{2}\right) q_{s}-\eta_{s} f\left(\eta_{s}\right)\right\}}{q_{s}^{2}}, \\
& \beta=\frac{f\left(\eta_{s}\right)\left\{f\left(\eta_{s}\right)-q_{s} \eta_{s}\right\}}{q_{s}^{2}},
\end{aligned}
$$

$$
\begin{aligned}
& \alpha_{s}=1+\ln \left(q_{s}\right)-\ln \left(q_{s}\right) \ln \left(-\ln q_{s}\right) \\
& \beta_{s}=\ln \left(q_{s}\right) \\
& \gamma_{E}=\frac{q_{s} \ln \left(q_{s}\right)\left\{q_{r s}\left[-1+\left(1+\ln \left(q_{s}\right)\right) \ln \left(-\ln \left(q_{s}\right)\right)\right]+q_{s} \ln \left(q_{s}\right) \ln \left(-\ln \left(q_{s}\right)\right)-q_{r} \ln \left(q_{r}\right) \ln \left(-\ln \left(q_{r}\right)\right)\right\}}{q_{r s}^{2}} \\
& \rho_{E}=\frac{-q_{s} \ln \left(q_{s}\right)\left[\left(1+\ln \left(q_{s}\right)\right) q_{r s}+q_{s} \ln \left(q_{s}\right)\right]}{q_{r s}^{2}}
\end{aligned}
$$

$$
\begin{aligned}
h_{1}\left(z_{r: n}, Z_{s: n}\right) & =\frac{f\left(Z_{s: n}\right)}{F\left(Z_{s: n}\right)-F\left(Z_{s: n}\right)} \\
& =\gamma_{E}+\rho_{E} z_{r: n}+v_{E} Z_{s: n} .
\end{aligned}
$$

The above constants can be taken to be and

$$
v_{E}=\frac{q_{s} \ln \left(q_{s}\right) q_{r} \ln \left(q_{r}\right)}{q_{r s}^{2}},
$$

where $q_{i j}=q-q_{j}$.

C.

See Algorithm 1.

\section{Data Availability}

Data in examples of this study are cited from reference papers. We have put citation in each example and listed cited papers in references.

\section{Conflicts of Interest}

The authors declare that there are no conflicts of interest regarding the publication of this paper. 


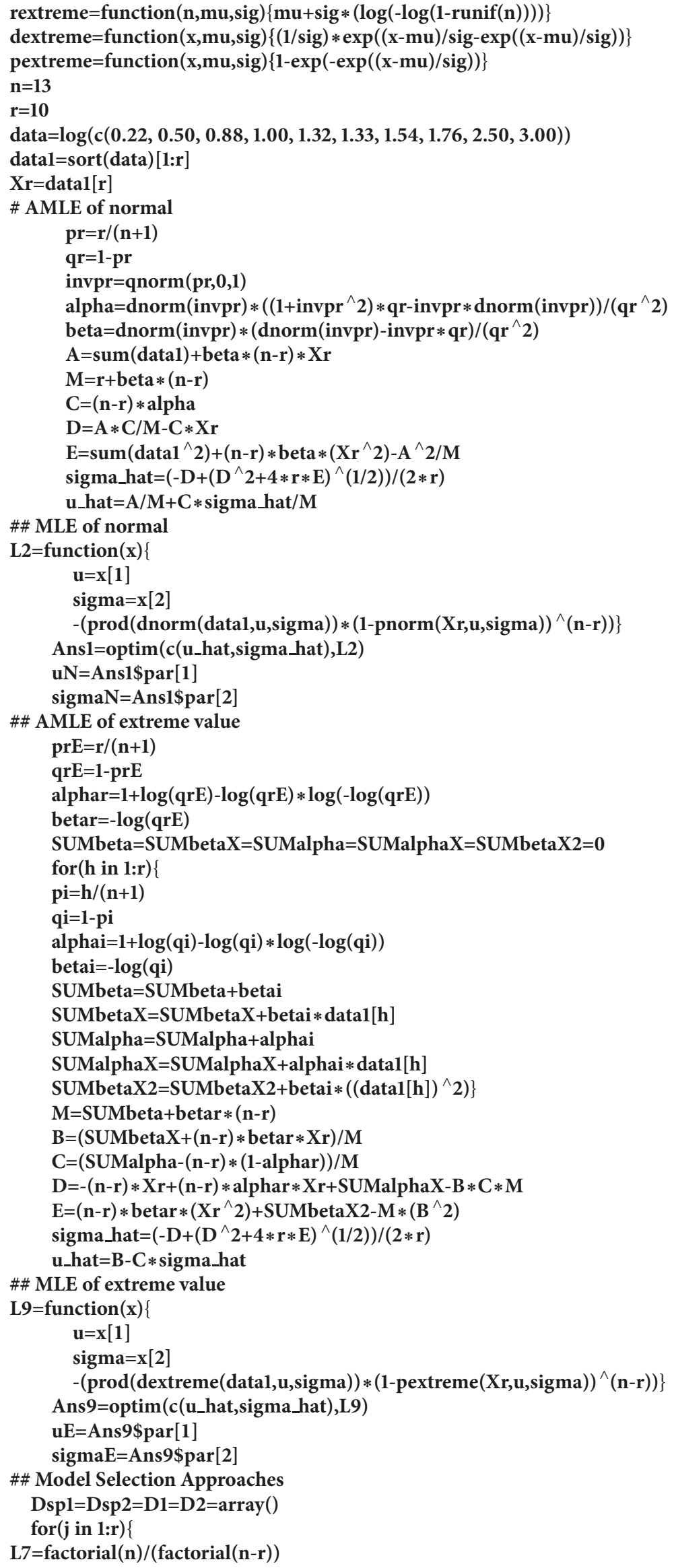




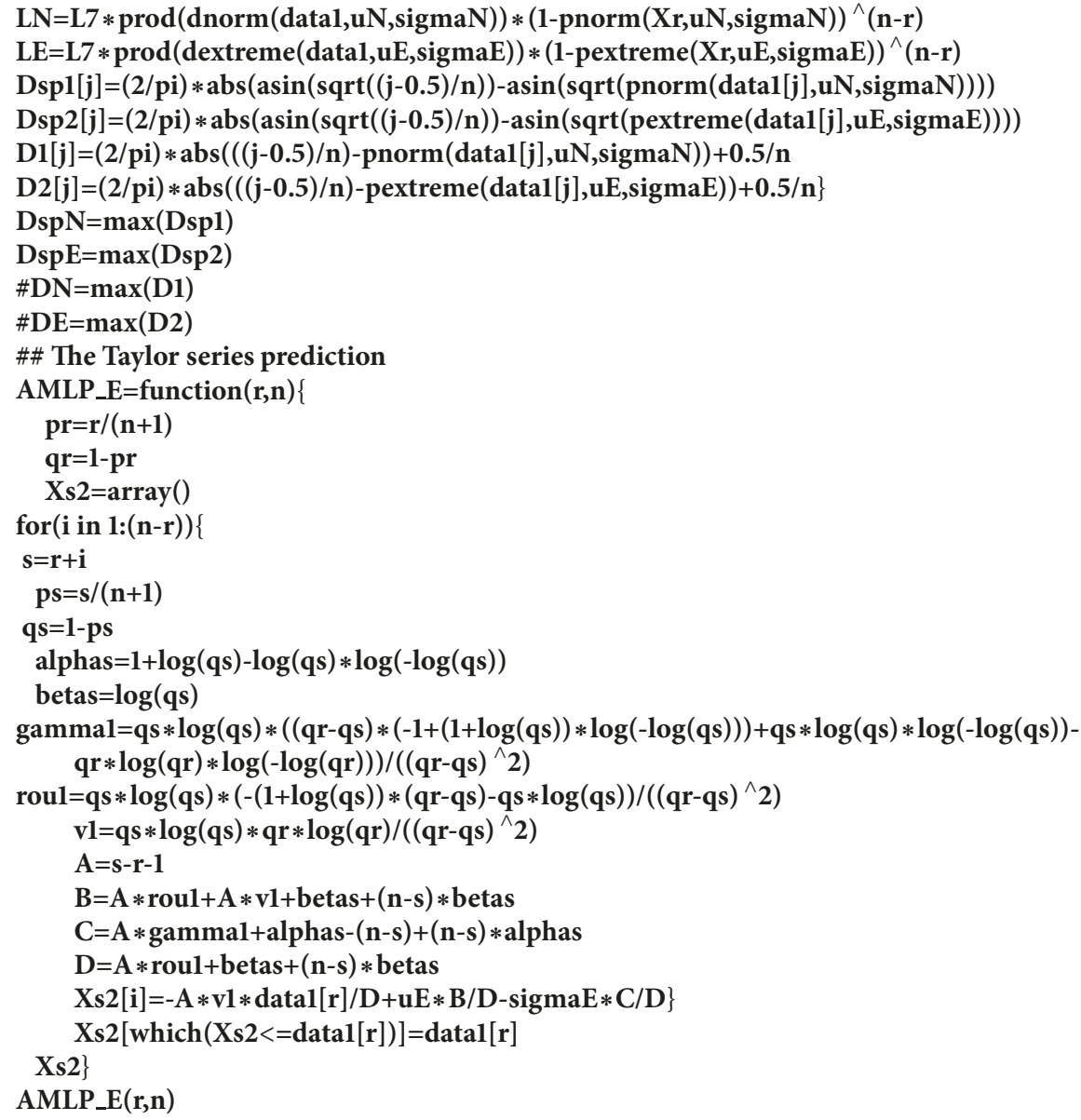

Algorithm 1: R code of Example 1.

\section{References}

[1] W. Q. Meeker Jr., "A comparison of accelerated life test plans for Weibull and lognormal distributions and type-I censoring," Technometrics, vol. 26, no. 2, pp. 157-171, 1984.

[2] Y. Dai, Y. F. Zhou, and Y. Z. Jia, "Distribution of time between failures of machining center based on type I censored data," Reliability Engineering \& System Safety, vol. 79, no. 3, pp. 377379, 2003.

[3] R. Sundberg, "Comparison of confidence procedures for type I censored exponential lifetimes," Lifetime Data Analysis. An International Journal Devoted to Statistical Methods and Applications for Time-to-Event Data, vol. 7, no. 4, pp. 393-413, 2001.

[4] N. Ahmad and A. Islam, "Optimal accelerated life test designs for Burr type XII distributions under periodic inspection and type I censoring," Naval Research Logistics (NRL), vol. 43, no. 8, pp. 1049-1077, 1996.

[5] G. K. Bhattacharyya, "The asymptotics of maximum likelihood and related estimators based on type II censored data," Journal of the American Statistical Association, vol. 80, no. 390, pp. 398404, 1985.

[6] T.-R. Tsai, J.-Y. Chiang, T. Liang, and M.-C. Yang, "Efficient Bayesian sampling plans for exponential distributions with
type-I-censored samples," Journal of Statistical Computation and Simulation, vol. 84, no. 5, pp. 964-981, 2014.

[7] K. S. Kaminsky and P. I. Nelson, "Prediction of order statistics," in Balakrishnan, N. Balakrishnan and R. C. Rao, Eds., pp. 431450, Handbook of Statistics 17, Order Statistics: Applications, New York, NY, USA, 1998.

[8] K. W. Fertig, M. E. Meyer, and N. R. Mann, “On constructing prediction intervals for samples from a weibull or extreme value distribution," Technometrics, vol. 22, no. 4, pp. 567-573, 1980.

[9] K. S. Kaminsky and L. S. Rhodin, "Maximum likelihood prediction," Annals of the Institute of Statistical Mathematics, vol. 37, no. 3, pp. 507-517, 1985.

[10] J.-W. Wu, H.-L. Lu, C.-H. Chen, and -H. Yang, "A note on the prediction intervals for a future ordered observation from a Pareto distribution," Quality \& Quantity, vol. 38, pp. 217-233, 2004.

[11] D. Kundu and M. Z. Raqab, "Bayesian inference and prediction of order statistics for a Type-II censored Weibull distribution," Journal of Statistical Planning and Inference, vol. 142, no. 1, pp. 41-47, 2012.

[12] H. Panahi and A. Sayyareh, "Parameter estimation and prediction of order statistics for the Burr type XII distribution with type II censoring," Journal of Applied Statistics, vol. 41, no. 1, pp. 215-232, 2014. 
[13] M. Z. Raqab, "Modified maximum likelihood predictors of future order statistics from normal samples," Computational Statistics \& Data Analysis, vol. 25, no. 1, pp. 91-106, 1997.

[14] C.-H. Yang and L.-I. Tong, "Predicting type II censored data from factorial experiments using modified maximum likelihood predictor," The International Journal of Advanced Manufacturing Technology, vol. 30, no. 9-10, pp. 887-896, 2006.

[15] J.-Y. Chiang, "Modified maximum likelihood prediction for type II censored data under the Weibull distribution," International Journal of Intelligent Technologies and Applied Statistics, vol. 3, no. 1, pp. 17-32, 2010.

[16] R. Dumonceaux and C. E. Antle, "Discrimination between the log-normal and the weibull distributions," Technometrics, vol. 15, no. 4, pp. 923-926, 1973.

[17] D. Kundu and A. Manglick, "Discriminating between the lognormal and gamma distributions," Journal of Applied Statistical Science, vol. 14, no. 1-2, pp. 175-187, 2005.

[18] D. Kundu and M. Z. Raqab, "Discriminating between the generalized Rayleigh and log-normal distribution," Statistics. A Journal of Theoretical and Applied Statistics, vol. 41, no. 6, pp. 505-515, 2007.

[19] H.-F. Yu, "Mis-specification analysis between normal and extreme value distributions for a screening experiment," Computers \& Industrial Engineering, vol. 56, no. 4, pp. 1657-1667, 2009.

[20] A. K. Dey and D. Kundu, "Discriminating between the lognormal and log-logistic distributions," Communications in Statistics-Theory and Methods, vol. 39, no. 1-2, pp. 280-292, 2010.

[21] A. E. Elsherpieny, N. S. Ibrahim, and U. N. Radwan, "Discriminating between Weibull and log-logistic distributions," International Journal of Innovative Research in Science, Engineering and Technology, vol. 2, no. 8, pp. 3358-3371, 2013.

[22] S. K. Ashour and A. M. Hashish, "A numerical comparison of three procedures used in failure model discrimination," Pakistan Journal of Statistics and Operation Research, vol. 10, no. 1, pp. 107-119, 2014.

[23] R. Pakyari, "Discriminating between generalized exponential, geometric extreme exponential and Weibull distributions," Journal of Statistical Computation and Simulation, vol. 80, no. 12, pp. 1403-1412, 2010.

[24] E. A. Elsherpieny, H. Z. Muhammed, and N. U. Mohamed Mohamed Radwan, "On discriminating between gamma and log-logistic distributions in case of progressive type II censoring," Pakistan Journal of Statistics and Operation Research, vol. 13, no. 1, pp. 157-183, 2017.

[25] A. Hossain and A. R. Willan, "Approximate MLEs of the parameters of location-scale models under type II censoring," Statistics. A Journal of Theoretical and Applied Statistics, vol. 41, no. 5, pp. 385-394, 2007.

[26] K. G. Mehrotra and P. Nanda, "Unbiased estimation of parameters by order statistics in the case of censored samples," Biometrika, vol. 61, pp. 601-606, 1974.

[27] N. Balakrishnan and A. C. Cohen, Order statistics and inference, Statistical Modeling and Decision Science, Academic Press, Inc., Boston, MA, 1991.

[28] D. Teichroew, "Tables of expected values of order statistics and products of order statistics for samples of size twenty and less from the normal distribution," Annals of Mathematical Statistics, vol. 27, pp. 410-426, 1956.
[29] C. Castro-Kuriss, D. M. Kelmansky, V. Leiva, and E. J. Martizez, "A new goodness-of-fit test for censored data with an application in monitoring processes," Communications in Statistics-Simulation and Computation, vol. 38, no. 6-7, pp. 1161-1177, 2009.

[30] N. R. Mann and K. W. Fertig, "Tables for obtaining Weibull confidence bounds and tolerance bounds based on best linear invariant estimates of parameters of the extreme-value distribution," Technometrics. A Journal of Statistics for the Physical, Chemical and Engineering Sciences, vol. 15, pp. 87-101, 1973.

[31] J. Lieblein and M. Zelen, "Statistical investigation of the fatigue life of deep-groove ball bearings," Journal of Research of the National Bureau of Standards, vol. 57, no. 5, pp. 273-316, 1956.

[32] W. Q. Meeker and L. A. Escobar, Statistical Methods for Reliability Data, John Wiley and Sons, New York, NY, USA, 1998.

[33] D. M. Byrne and S. Taguchi, "Taguchi approach to parameter design,” Quality Progress, vol. 20, no. 12, pp. 19-26, 1987. 


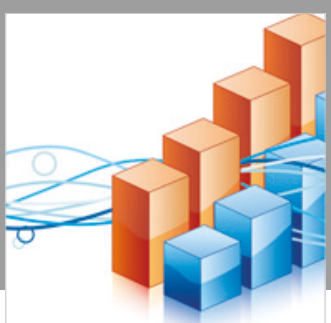

Advances in

Operations Research

\section{-n-m}
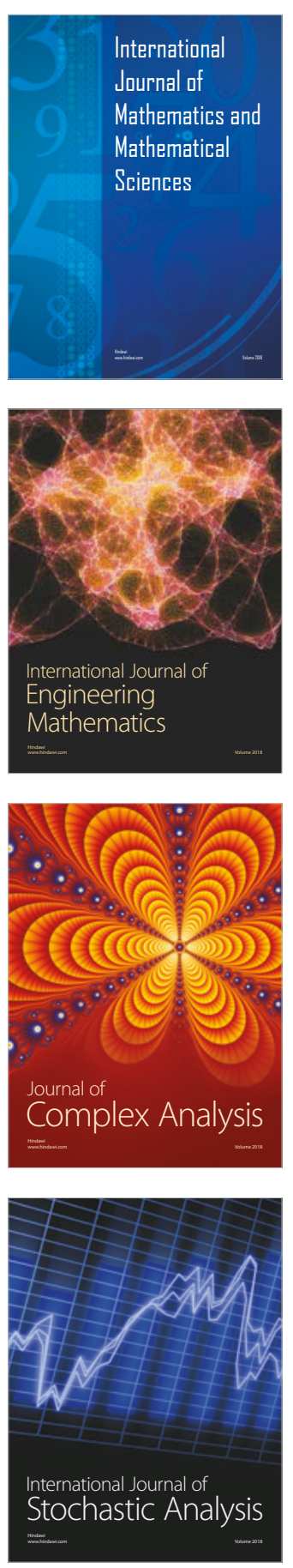
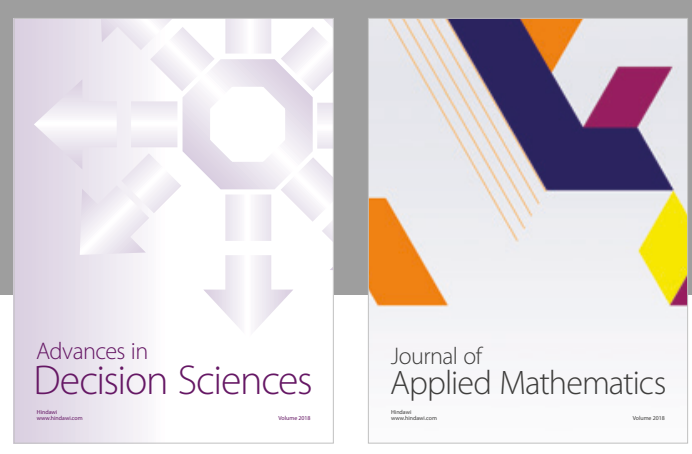

Journal of

Applied Mathematics
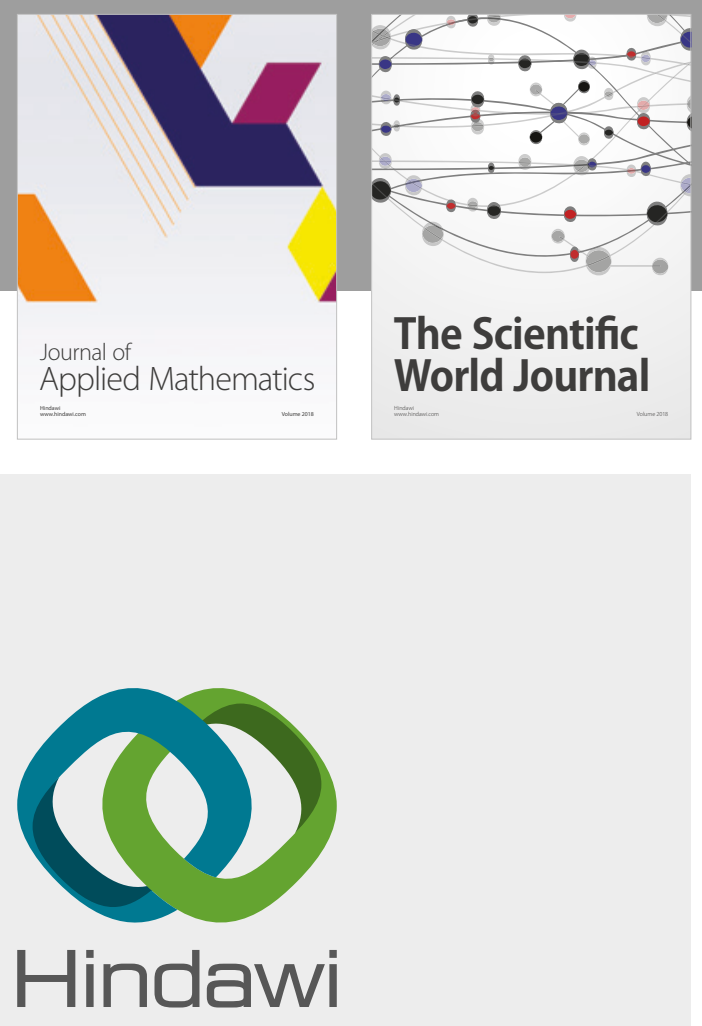

Submit your manuscripts at

www.hindawi.com

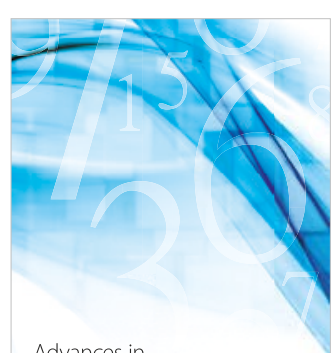

Advances in
Numerical Analysis
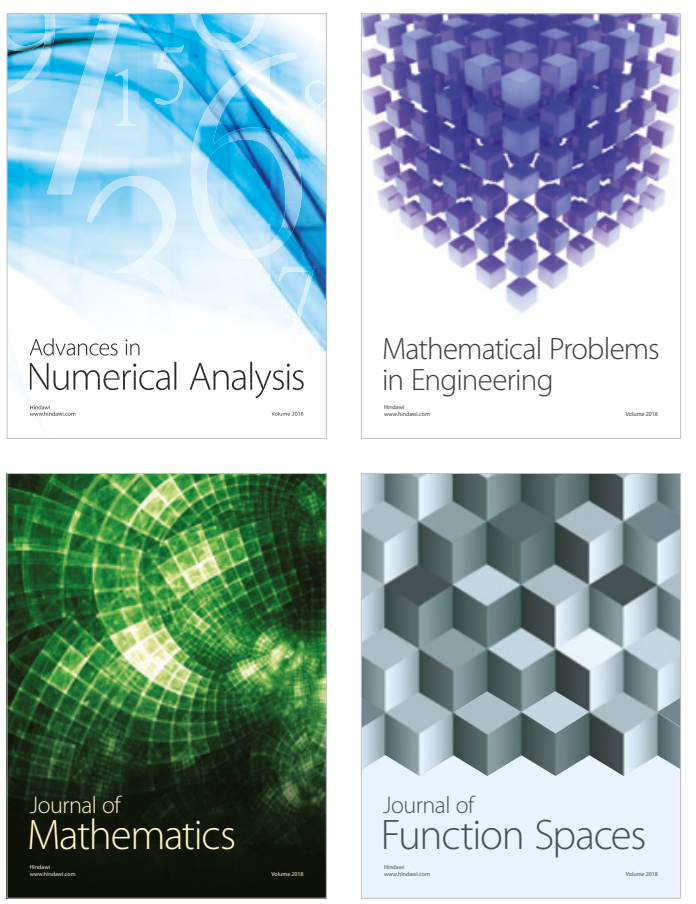

Mathematical Problems in Engineering

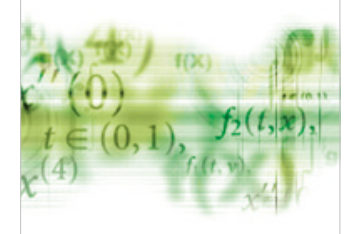

International Journal of

Differential Equations

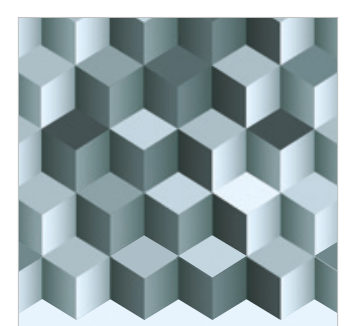

Journal of

Function Spaces

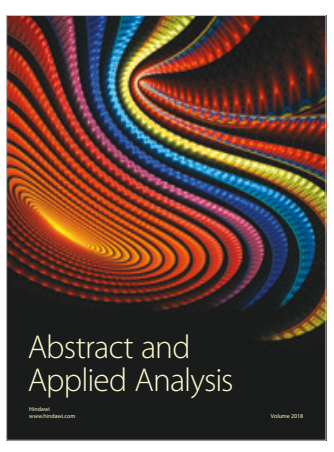

The Scientific

World Journal

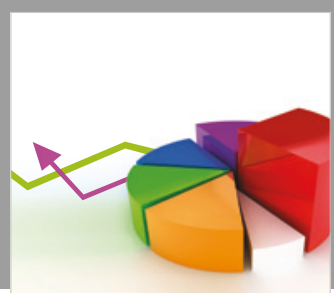

Journal of

Probability and Statistics
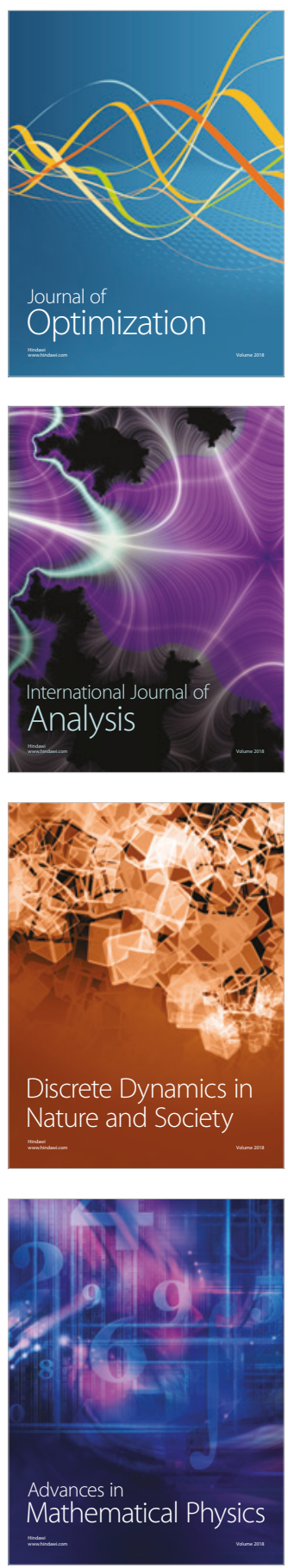